\title{
OPTIMAL NATURAL DUALITIES
}

\author{
B. A. DAVEY AND H. A. PRIESTLEY
}

This work is dedicated to the memory of Alan Day

\begin{abstract}
The authors showed previously that for each of the varieties $\mathbf{B}_{n}(3 \leq$ $n<\omega)$ of pseudocomplemented distributive lattices there exists a natural duality given by a set of $p(n)+3$ binary algebraic relations, where $p(n)$ denotes the number of partitions of $n$. This paper improves this result by establishing that an optimal set of $n+3$ of these relations suffices. This is achieved by the use of "test algebras": it is shown that redundancy among the relations of a duality for a prevariety generated by a finite algebra may be decided by testing the duality on the relations, qua algebras.
\end{abstract}

\section{INTRODUCTION}

This paper opens a new chapter in duality theory. It was inspired by our companion paper [4], in which we established natural dualities for the subvarieties $\mathbf{B}_{n}(n \leq n<\omega)$ of the variety $\mathbf{B}_{\omega}$ of distributive $p$-algebras. The schizophrenic object defining the duality for $\mathbf{B}_{n}$ involved relations whose number increases exponentially with $n$ and we were led to ask whether a more tractable duality exists. Until now the detection of redundancy among relations in a duality has been more an art than a science, and consequently a daunting task. By introducing "test algebras" we show that science can supplant art. Our new technique enables us to obtain a significantly simplified duality for $\mathbf{B}_{n}$ in case $n>3$, to show that, in a very strong sense, no simplification is possible for $n \leq 3$, and to derive an optimality theorem valid for every $n$. To explain the test algebra technique (which has potential value well beyond that exhibited by the applications given here) we first need to set up some of the duality framework constructed by Davey and Werner in [5].

Consider the prevariety $\mathscr{A}=\mathbb{I S P}(\underline{P})$ generated by a finite algebra $\underline{P}$. A $k$ ary relation $r$ on $P$ is called algebraic if it is a subalgebra of $\underline{P}^{k}$. Given a family $R$ of algebraic relations on $\underline{P}$ we define a topological, relational structure $P:=$ $(P, \mathscr{T}, R)$, where $\mathscr{T}$ is the discrete topology. Define $\mathscr{X}$ to be the category of closed substructures of powers of $\underset{P}{P}$. Given $A \in \mathscr{A}$, each relation $r \in R$ may be extended pointwise to the set $\mathscr{A}(A, \underline{P})$ of homomorphisms from $A$ into $\underline{P}$. It is then easily seen that $\mathscr{A}(A, \underline{P})$ is a closed substructure of $\underline{P}^{A}$. For each $A \in \mathscr{A}$ and all $a \in A$, the evaluation map $e_{A}(a): \mathscr{A}(A, \underline{P}) \rightarrow \underline{P}$, given by $\varphi \mapsto$

Received by the editors April 30, 1991.

1991 Mathematics Subject Classification. Primary 06D15, 06D05, 08B99, 05A17, 18A40.

Key words and phrases. Distributive p-algebra, natural duality, piggyback duality, optimal duality. 
$\varphi(a)$, is continuous and preserves each relation $r \in R$. If the evaluation maps are the only continuous relation-preserving maps from $\mathscr{A}(A, \underline{P})$ to $\underline{P}$, then we have a representation of $A$ as an algebra of continuous relation-preserving riaps. In this case we say that $R$ (or $P$ ) yields a duality on $A$. If $R$ yields a duality on each algebra $A \in \mathscr{A}$, then we say that $R$ (or $P$ ) yields a duality on $\mathscr{A}$. All of this may be dressed up in appropriately categorical language. We have well-defined contravariant hom-functors

$$
D(-):=\mathscr{A}(-, \underline{P}): \mathscr{A} \rightarrow \mathscr{X}, \quad \text { and } \quad E(-):=\mathscr{X}(-, \underline{P}): \mathscr{X} \rightarrow \mathscr{A} .
$$

If $R$ yields a duality on $\mathscr{A}$, then $\mathscr{A}$ is dually equivalent to a subcategory of $\mathscr{X}$ via the functors $D$ and $E$. (See Lemmas 1.1 to 1.5 in [5] for the details.) In summary we see that if the alter ego $P=(P ; \mathscr{T}, R)$ of $\underline{P}$ is suitably chosen, then we have a concrete representation of each algebra $A \in \mathscr{A}$ as the algebra $E D(A)$ of all continuous, $R$-preserving maps from $D(A)$ to $P$.

It is a fundamental tenet of [5] that the relations in $R$ be algebraic. This assumption underpins the categorical formalism presented above, since it is exactly what is needed to ensure that the functors $D$ and $E$ are well defined. Thus the algebraicity of the relations in $R$ might be considered as already having served its purpose. In fact we shall exploit this assumption in another way, by taking advantage of an extension of the schizophrenia inherent in the $\underline{P}$ versus $\underline{P}$ personality split. Each relation $r \in R$ lives a second life as a subalgebra of some $\underline{P}^{k}$; we shall denote this algebra by $\underline{r}$. Thus $\underline{r} \in \mathscr{A}$.

Assume that we have a finite set $R$ of algebraic relations on $\underline{P}$ which yields a duality on $\mathscr{A}$. In practice we would like $R$ to be minimal with respect to yielding a duality on $\mathscr{A}$, and preferably also of the minimum possible size. Fix $r \in R$ and consider $R^{*}:=R \backslash\{r\}$. It is not at all clear that the problem of determining whether $R^{*}$ also yields a duality on $\mathscr{A}$ is a finite one since it apparently requires us to check that $R^{*}$ yields a duality on every algebra $A \in \mathscr{A}$. In $\S 2$ we prove (with surprising ease) a surprising result. We establish that $R^{*}$ yields a duality on $\mathscr{A}$ provided it yields a duality on a single algebranamely the algebra $\underline{r}$ (which we shall henceforth refer to as the test algebra corresponding to the relation $r$ ). We therefore have an algorithm for deciding whether $R^{*}$ yields a duality on $\mathscr{A}$. This involves two steps.

(1) Obtain a viable description of $D(\underline{r})$, that is, of $\mathscr{A}(\underline{r}, \underline{P})$ and the relational structure on it induced by $P:=\left(P ; \mathscr{T}, R^{*}\right)$.

(2) Compute the number, $N=|E D(\underline{r})|$, of $R^{*}$-preserving maps from $D(\underline{r})$ to $\underset{\sim}{P}$ with $|r|$.

Proposition 2.3 implies that $r$ can be deleted from $R$ without destroying the duality if and only if $N \ngtr|\underline{r}|$. It follows that the problem of reducing $R$ to a minimal family is one which, with sufficient computer power and human cunning, can be programmed.

Before we can explain what is involved in applying the above procedure to the varieties $\mathbf{B}_{n}$, some recapitulation is necessary.

Recall that $\mathbf{B}_{\omega}$ is the class of algebras $\left(A ; \vee, \wedge,{ }^{*}, 0,1\right)$ of type $(2,2,1$, $0,0)$ such that $(A ; \vee, \wedge, 0,1)$ is a bounded distributive lattice and $a^{*}$ (the pseudocomplement of $a$ ) is given by $a^{*}=\max \{b \in A \mid a \wedge b=0\}$. The lattice of subvarieties of $\mathbf{B}_{\omega}$ is an $\omega+1$ chain

$$
\mathbf{B}_{-1} \subset \mathbf{B}_{0} \subset \mathbf{B}_{1} \subset \cdots \subset \mathbf{B}_{n} \subset \cdots \subset \mathbf{B}_{\omega},
$$


where $\mathbf{B}_{-1}$ is the trivial variety, $\mathbf{B}_{0}$ is the variety of Boolean algebras and $\mathbf{B}_{1}$ is the variety of Stone algebras. For $n \geq 0$, we have $\mathbf{B}_{n}=\operatorname{ISP}\left(\underline{P}_{n}\right)$ where the $p$-algebra $\underline{P}_{n}$ is obtained by adjoining a new top, T, to the $n$-atom Boolean lattice (whose elements we identify with subsets of $\{1,2, \ldots, n\}$, with $\varnothing$ designated $\perp$ and $\{1,2, \ldots, n\}$ designated $d$ ).

To obtain a duality for $\mathbf{B}_{n}$ following the Davey-Werner pattern it is necessary to define a suitable topological, relational structure $P_{n}=\left(P_{n} ; \mathscr{T}, R\right)$ on the underlying set $P_{n}$ of $\underline{P}_{n}$. This was achieved for $n=0$ (Boolean algebras) by Stone [12] and, 40 years later, for $n=1$ (Stone algebras) by Davey [1, 2]. Higher values of $n$ remained out of reach until Davey and Werner's piggyback technique $[6,7]$ became available; this was successfully applied to $\mathbf{B}_{2}$ in $[6,7]$, and, with additional invocation of duality methods, extended to arbitrary $\mathbf{B}_{n}$ in [4]. For $n=0$ the set of relations is empty. For each $n>0$, the duality is given by a suitable set $R$ of binary algebraic relations on $\underline{P}_{n}$. We give a full description of $R$ (as defined in [4]) when we have the necessary notation to hand (see $\S 3$ ). Here it suffices to recall some salient points. We may write $R=S \cup G$ where

(i) $S$ is a set of binary algebraic relations in one-to-one correspondence with the partitions of the integer $n$, and

(ii) $G$ is a generating set for the endomorphism monoid, End $\underline{P}_{n}$, of $\underline{P}_{n}$. (Here we have blurred the distinction between an endomorphism and its graph.) The endomorphisms of $\underline{P}_{n}$ are of two types:

(a) automorphisms, each of which is induced by a permutation of the atoms $\{1\},\{2\},, \ldots,\{n\}$ of $\underline{P}_{n}$;

(b) endomorphisms with image $\{\perp, T\}$, each of which is a map $e_{i}(i \in$ $\{1,2, \ldots, n\})$ where $e_{i}^{-1}(\perp)$ consists of those subsets of $\{1,2, \ldots, n\}$ which do not contain $i$.

We denote by $f_{\delta}$ and $f_{\gamma}$ the automorphisms induced by, respectively, (12) and $(123)$, and let $f_{\nu}:=e_{1}$. Then End $\underline{P}_{n}$ is generated by $f_{\nu}(n=1)$, by $f_{\nu}, f_{\delta}(n=2)$, and by $f_{\nu}, f_{\delta}, f_{\gamma}(n \geq 3)$.

Thus, for every $n$ we have a duality for $\mathbf{B}_{n}$ involving at most $p(n)+3$ binary relations, where $p(n)$ is the number of partitions of the integer $n$. Table 1 specifies the dualities for $n \leq 3$. The relations in $S$ are depicted, for $n=$ $1,2,3$, in Figures 1, 2, 3, respectively. (To save space, we have not drawn $\leq$ in the case $n=3$. It suffices to know that for all $n \geq 1$ the order is an antichain except for the relation $d<T$.) These relations are induced by partitions as follows: the order $\leq$ arises from the $n$-part partition $(1,1, \ldots, 1)$, the relation $\dashv$ from the 1-part partition, and the relation $\triangleleft$ from the unique 2-part partition $(2,1)$ of the integer 3 . For an explanation of this correspondence, see [4].

TABLE 1

\begin{tabular}{|c|c|c|c|}
\hline Variety & Endomorphisms & Relations & References \\
\hline $\mathbf{B}_{0}$ & $\varnothing$ & $\varnothing$ & {$[12]$} \\
\hline $\mathbf{B}_{1}$ & $f_{\nu}$ & $\leq$ & {$[1,2]$} \\
\hline $\mathbf{B}_{2}$ & $f_{\nu}, f_{\delta}$ & $\leq, \dashv$ & {$[6,7]$} \\
\hline $\mathbf{B}_{3}$ & $f_{\nu}, f_{\delta}, f_{\gamma}$ & $\leq, \dashv, \triangleleft$ & {$[4]$} \\
\hline
\end{tabular}




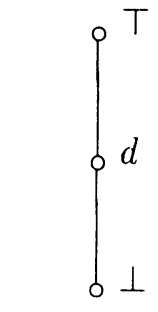

$\underline{P}_{1}$

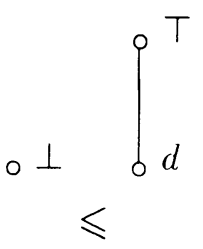

FiguRe 1

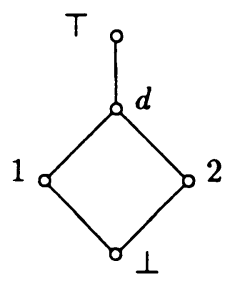

$\underline{P}_{2}$

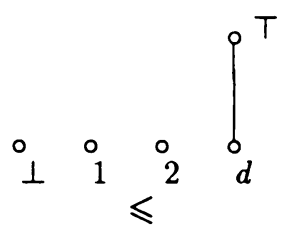

FigURe 2

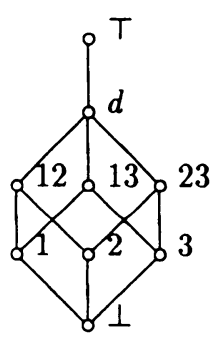

$\underline{P}_{3}$

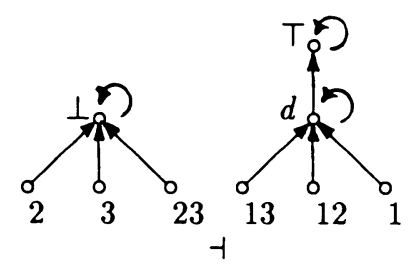

FIGURE 3
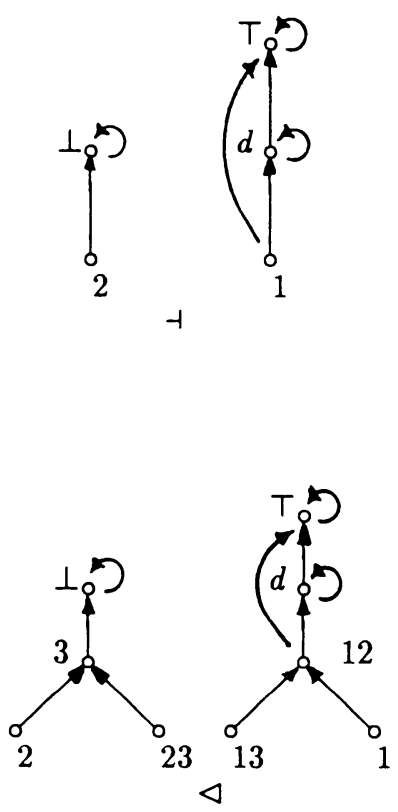

For $n=1,2,3$, the duality for $\mathbf{B}_{n}$ involves $n$ nonendomorphisms, and $n$ equals $p(n)$. As $n=4$ is the smallest value such that $p(n)>n$, it was clear that careful analysis of the cases $n=3$ and $n=4$ would be required if we were to decide whether the minimal number of nonendomorphisms needed in general is closer to $n$ or to the inherently exponential $p(n)$. This was our stepping-off point for the present paper. We sought to discover whether the number of partition-induced relations in the duality for $\mathbf{B}_{4}$ could be reduced from $p(4)$ (viz. 5) to 4.

The idea of using a "test algebra" to discriminate between avoidable and unavoidable relations goes back to this very early stage of the project, though the theory presented in $\S 2$ evolved much later. Even without that theory, it was trivial that if we could find an algebra $A \in \mathbf{B}_{n}$ such that $R$ yielded a duality on $A$ but $R \backslash\{r\}$ failed to do so, then $r$ could not be dropped without destroying the duality. It quickly became clear that the most tractable choices for $A$, for example short chains, were not rich enough in structure to yield information. 
The next obvious candidate for $A$ was the algebra $\underline{r}$. Taking $\underline{r}$ as the test algebra, for each $r \in R$ in turn, and calculating by hand we were able to show that none of the relations $\leq, \dashv$, and $\triangleleft$ could be dropped in the case $n=3$. For $n=4$ there were two obvious candidates for deletion: the relations corresponding to the partitions $(2,2)$ and $(3,1)$ have isomorphic test algebras and hence it was natural to try to delete one of these relations (which we shall call $r^{\prime}$ and $\left.r^{\prime \prime}\right)$. Up to isomorphism we have

$$
\underline{r}^{\prime}=\underline{r}^{\prime \prime}=\left(\left(\mathbf{2}^{2} \oplus \mathbf{1}\right) \times \mathbf{2}^{2}\right) \oplus \mathbf{1} .
$$

Hence if $r$ denotes either $r^{\prime}$ or $r^{\prime \prime}$, then $|\underline{r}|=21$. Also $\left|P_{4}\right|=17$ and $|R \backslash\{r\}|=7$. The theory which we developed to handle Step (1) of the test algebra procedure for $\mathbf{B}_{n}$ (and which is presented in $\S 3$ ) told us that $\mathbf{B}_{4}\left(\underline{r}, \underline{P}_{4}\right)$ has 42 elements and provided easy access to its relational structure. We were then faced with the intimidating task of calculating the number of maps from a 42-element set into a 17-element set which preserve 7 relations.

At this juncture we sought computer assistance. We are grateful to Dr. Martin Ward for writing a viable program (which appears in [13]) for calculating the relation-preserving maps between two finite sets each carrying a finite set of binary relations. His iterative backtracking algorithm, refined in various ways specific to the $\mathbf{B}_{n}$ problem, allowed us to investigate the case $n=4$ using only the computing power of a PC. We remark that the same program has subsequently provided valuable computer backup to the solution of a number of other problems involving relation-preserving maps and, in particular, problems concerning dualities; see [11].

Using the backtracking program we obtained the following results concerning the partition-induced duality for $\mathbf{B}_{4}$ :

(i) each of $R \backslash\left\{r^{\prime}\right\}$ and $R \backslash\left\{r^{\prime \prime}\right\}$ yields a duality on the common test algebra corresponding to the relations $r^{\prime}$ and $r^{\prime \prime}$ associated with the partitions $(2,2)$ and $(3,1)$;

(ii) $R \backslash\left\{r^{\prime}, r^{\prime \prime}\right\}$ fails to yield a duality on the test algebra corresponding to $r^{\prime}$ and $r^{\prime \prime}$;

(iii) when $r$ is the relation corresponding to any one of the partitions $(1,1$, $1,1),(2,1,1)$ and (4) then $R \backslash\{r\}$ fails to yield a duality on $\underline{r}$.

We deduced that the only possible reduction would be the removal of just one of the relations $r^{\prime}, r^{\prime \prime}$. It was at this stage that we proved the results of $\S 2$. Consequently we were able to conclude that either $r^{\prime}$ or $r^{\prime \prime}$ can indeed be removed, so that the reduction from $p(n)$ nonendomorphisms to $n$ nonendomorphisms can be achieved in this case.

Our experiments with $n=3$ and $n=4$ suggested that a minimal partitioninduced duality for $\mathbf{B}_{n}$ must include at least one relation associated with an $l$-part partition of $n$, for each of the possible values $1,2, \ldots, n$ of $l$ (along with a generating set for End $\left.\underline{P}_{n}\right)$. It turns out that the relations induced by partitions $\left(j_{1}, \ldots, j_{l_{1}}\right)$ and $\left(k_{1}, \ldots, k_{l_{2}}\right)$ are isomorphic as algebras if and only if $l_{1}=l_{2}$. Accordingly we define two binary algebraic relations to have the same shape if they are isomorphic as algebras and we say that a binary algebraic relation on $\underline{P}_{n}$ has shape $l$ if it has the same shape as the relation induced by some $l$-part partition. Equivalently, a binary algebraic relation $r$ has shape $l$ if and only if $\underline{r}$ is isomorphic to $\left(\left(\mathbf{2}^{l} \oplus \mathbf{1}\right) \times \mathbf{2}^{n-l}\right) \oplus \mathbf{1}$. 
In $\S 4$ we prove that if $S$ is a set of partition-induced relations and $G$ is a set of endomorphisms such that $R=S \cup G$ yields a duality on $\mathbf{B}_{n}$, then $G$ generates End $\underline{P}_{n}$ as a monoid. The main result of $\S 4$ applies to arbitrary binary algebraic relations not just to the partition-induced ones. It states that for each $l$ with $1 \leq l \leq n$, the relations of shape $l$ are unavoidable in any duality given by binary algebraic relations. More precisely, if $S$ is a family of binary algebraic relations on $\underline{P}_{n}$ which, along with some set $G$ of endomorphisms of $\underline{P}_{n}$, yields a duality on $\mathbf{B}_{n}$, then for all $l$ with $1 \leq l \leq n$, the set $S$ includes at least one relation of shape $l$. We deduce that $|S| \geq n$. An immediate consequence is the strongest possible optimality result for the dualities given earlier for $\mathbf{B}_{1}, \mathbf{B}_{2}$, and $\mathbf{B}_{3}$. For $n \in\{1,2,3\}$, let $S_{n} \subseteq \mathbb{S}\left(\underline{P}_{n}^{2}\right)$ be given by $S_{1}=\{\leq\}, S_{2}=\{\leq,-1\}$, and $S_{3}=\{\leq,-1, \triangleleft\}$. If $S$ is a family of binary algebraic relations such that $R=S \cup G$ yields a duality on $\mathbf{B}_{n}$, for some subset $G$ of End $\underline{P}_{n}$, then, up to replacing a relation by its converse, $S$ contains $S_{n}$ as a subset.

For $n \geq 4$ we cannot hope for such a sharp optimality result since it is possible to find relations $r$ and $s$ which both have shape $l$ while $s$ is not the converse of $r$. Certainly, there is no a priori reason why a set of algebraic relations which is minimal with respect to yielding a duality on a prevariety $\mathscr{A}$ should be unique in any way. Nevertheless there is a strong optimality theorem for each of the varieties $\mathbf{B}_{n}$ for $n \geq 4$. Suppose that $R=S \cup G$ is a set of binary algebraic relations yielding a duality on $\mathbf{B}_{n}$, where $G$ is a subset of End $\underline{P}_{n}$ and $S$ is a set of partition-induced relations. The theorems stated in the preceding paragraph imply that $|R| \geq n+3$. Our computer analysis shows that the lower bound $n+3$ can be attained when $n=4$. In $\S 5$ we confirm that this remains valid for general $n$. Specifically we prove that if $S=\left\{r_{1}, \ldots, r_{n}\right\}$, where $r_{l}$ is any binary algebraic relation on $\underline{P}_{n}$ of shape $l(1 \leq l \leq n)$ and $G$ is the 3-element generating set for End $\underline{P}_{n}$ defined earlier, then $\underline{P}=\left(P_{n} ; \mathscr{T}, S \cup G\right)$ yields a duality on $\mathbf{B}_{n}$. It took an inordinately long time to find a proof of this result which both authors were willing to believe simultaneously. We trust that the series of lemmas which culminate in the theorem will cause less pain in the reading than they did in the writing.

The endomorphisms of $\underline{P}_{n}$ play a somewhat ambivalent role here. On one hand, an endomorphism $e$ may be viewed as a unary operation in the type of $P_{n}$; the corresponding unary operation on $D(A)=\mathbf{B}_{n}\left(A, \underline{P}_{n}\right)$ is then defined in the obvious way via composition. Alternatively, we may replace $e$ by its graph and hence regard $e$ as a binary relation in the type of $P_{n}$. As far as developing a duality is concerned, it does not matter which path we elect to follow: a map $\varphi: D(A) \rightarrow P_{n}$ preserves the unary operation $e$ if and only if it preserves the corresponding binary relation. In applications of duality theory it is often important to know that we have a full duality between the algebraic category $\left(\mathbf{B}_{n}\right.$ in our case) and the topological category $\mathscr{X}$, that is, that the evaluation map from $X$ to $D E(X)$ is an isomorphism for all $X \in \mathscr{X}$, or equivalently, that each object $X \in \mathscr{X}$ is isomorphic to $D(A)$ for some $A \in \mathbf{B}_{n}$. Note that $D(A)$ is closed under the action of End $\underline{P}_{n}$, while arbitrary closed subsets of powers of $P_{n}$ need not be. Thus, in order to achieve a full duality we must refrain from replacing an endomorphism by its graph. Consequently, we often write $\underline{P}_{n}=\left(P_{n} ; \mathscr{T}, G, S\right)$ rather than $\underline{P}_{n}=\left(P_{n} ; \mathscr{T}, S \cup G\right)$. 


\section{Avoidable Relations}

Once we have a finite set $R$ of algebraic relations which yields a duality on $\mathscr{A}=\operatorname{ISP}(\underline{P})$, we naturally wish to delete relations from $R$ until a family is obtained which is minimal with respect to yielding a duality on $\mathscr{A}$. In the past, this often difficult problem has been solved by a combination of inspired guess work and ad hoc techniques. It was enough to stop the authors of $[6,7]$ in their tracks while trying to obtain a workable duality for the variety $\mathbf{B}_{3}$. In this section we add a little more science to the pot of available techniques.

Consider a fixed relation $r \in R$. We shall prove the tantalizing fact that in order be sure that $R \backslash\{r\}$ still yields a duality on $\mathscr{A}$, it suffices to check that $R \backslash\{r\}$ yields a duality on a single algebra-namely the test algebra $\underline{r} \in \mathscr{A}$. It follows that the problem of reducing $R$ to a minimal family is a finite one which we may hope to be able to program. As we have already mentioned, it was the success of such a computer program in producing a minimal duality for the variety $\mathbf{B}_{4}$ which ultimately led to this paper.

The idea of taking a relation $r \subseteq A^{2}$ which is a subalgebra of $\underline{A}^{2}$, viewing it as an algebra $r$ in its own right and then imposing $r$ on it as a relation has been very successfully exploited in other parts of universal algebra. For example, the idea of viewing a congruence $\alpha \in \operatorname{Con} \underline{A}$ as an algebra $\underline{\alpha}$ and then looking at the congruence $\alpha \times \alpha$ on $\underline{\alpha}$ is fundamental to Gumm's geometric approach to the commutator in modular varieties; see [9].

Although most of what follows in this section is valid when $\underline{P}$ is a compact topological algebra, to simplify the presentation we shall continue to assume that $P$ is finite and its topology discrete.

We say that a family $R$ of relations generates a relation $r$ on an algebra $A \in \mathscr{A}$ (or on a family $\mathscr{B} \subseteq \mathscr{A}$ ) if whenever a continuous map $\varphi: D(A) \rightarrow P$ preserves each relation in $R$, it also preserves $r$ (for each $A \in \mathscr{B}$ ). Clearly, if $R$ yields a duality on $\mathscr{B}$ and $R \backslash\{r\}$ generates $r$ on $\mathscr{B}$, then the smaller set, $R \backslash\{r\}$, also yields a duality on $\mathscr{B}$. If $R$ generates $r$ on $\mathscr{A}$, then we say simply that $R$ generates $r$.

We begin with a simple lemma from which the main results of this section follow. To avoid confusion, we shall sometimes denote a relation $r$ on $P$ by $r_{P}$ and its pointwise extension to $D(A)=\mathscr{A}(A, \underline{P})$ by $r_{D(A)}$. If $f_{1}, \ldots, f_{n} \in$ $D(A)$, then the product map $f_{1} \sqcap \cdots \sqcap f_{n}: A \rightarrow P^{n}$ is defined by

$$
(\forall a \in A)\left(f_{1} \sqcap \cdots \sqcap f_{n}\right)(a):=\left(f_{1}(a), \ldots, f_{n}(a)\right) .
$$

Observe that $\left(f_{1}, \ldots, f_{n}\right) \in r_{D(A)}$ says precisely that the image of the homomorphism $f_{1} \sqcap \cdots \sqcap f_{n}$ lies in the subalgebra $\underline{r}$ of $\underline{P}^{n}$. Hence, if $\left(f_{1}, \ldots, f_{n}\right) \in$ $r_{D(A)}$, then $f_{1} \sqcap \cdots \sqcap f_{n}: A \rightarrow \underline{r}$ is a well-defined homomorphism.

Lemma 2.1. Let $A \in \mathscr{A}$, let $\underline{r} \leq \underline{P}^{n}$, and let $\varphi: D(A) \rightarrow P$ be a map. If $\left(f_{1}, \ldots, f_{n}\right) \in r_{D(A)}$ and $\varphi \circ D\left(f_{1} \sqcap \cdots \sqcap f_{n}\right): D(\underline{r}) \rightarrow P$ is given by evaluation at some point $c \in r$, then $\left(\varphi\left(f_{1}\right), \ldots, \varphi\left(f_{n}\right)\right) \in r_{P}$.

Proof. Assume that $\varphi \circ D\left(f_{1} \sqcap \cdots \sqcap f_{n}\right)=e_{\underline{r}}(c)$ for some $c=\left(c_{1}, \ldots, c_{n}\right) \in r$. Let $\pi_{i}: r \rightarrow P$ be the $i$ th projection. Then

$$
\begin{aligned}
\varphi\left(f_{i}\right) & =\varphi\left(\pi_{i} \circ\left(f_{1} \sqcap \cdots \sqcap f_{n}\right)\right)=\varphi\left(D\left(f_{1} \sqcap \cdots \sqcap f_{n}\right)\left(\pi_{i}\right)\right) \\
& =\left(e_{\underline{r}}(c)\right)\left(\pi_{i}\right)=\pi_{i}(c)=c_{i},
\end{aligned}
$$

and consequently $\left(\varphi\left(f_{1}\right), \ldots, \varphi\left(f_{n}\right)\right)=\left(c_{1}, \ldots, c_{n}\right)=c \in r_{P}$. 
While this lemma may look a little technical, its consequences are both simply stated and somewhat surprising.

Proposition 2.2. Let $R$ be a family of finitary algebraic relations on $\underline{P}$. If $R$ yields a duality on the test algebra $\underline{r} \leq \underline{P}^{n}$, then $R$ generates $r$.

Proof. Let $A \in \mathscr{A}$ and assume that $\varphi: D(A) \rightarrow P$ is continuous and preserves the relations in $R$. We must show that $\varphi$ preserves $r$. Let $f_{1}, \ldots, f_{n} \in D(A)$ with $\left(f_{1}, \ldots, f_{n}\right) \in r_{D(A)}$. Since $\psi:=\varphi \circ D\left(f_{1} \sqcap \cdots \sqcap f_{n}\right): D(\underline{r}) \rightarrow P$ is well defined, continuous and preserves the relations in $R$ and since $R$ yields a duality on $\underline{r}$, it follows that $\psi=e_{\underline{r}}(c)$ for some $c \in r$. Thus, by Lemma 2.1, we have $\left(\varphi\left(f_{1}\right), \ldots, \varphi\left(f_{n}\right)\right) \in r_{P}$, as required.

Proposition 2.3. Let $R_{1}$ be a family of finitary algebraic relations on $\underline{P}$. Let $R$ be a subset of $R_{1}$ and assume that $R_{1}$ yields a duality on $\mathscr{A}$. Then the following are equivalent:

(i) $R$ yields a duality on $\mathscr{A}$.

(ii) $R$ yields a duality on each of the test algebras $\underline{r}$ where $r \in R_{1} \backslash R$.

(iii) $R$ generates each of the deleted relations in $R_{1} \backslash R$ on each of the test algebras $\underline{r}$ where $r \in R_{1} \backslash R$.

Proof. Since (i) $\Rightarrow$ (ii) $\Leftrightarrow$ (iii) is clear, only (ii) $\Rightarrow$ (i) remains to be proved. Since $R_{1}$ yields a duality on $\mathscr{A}$, to prove that $R$ also yields a duality on $\mathscr{A}$ it suffices to show that $R$ generates each relation in $R_{1} \backslash R$. Given that $R$ yields a duality on each of the test algebras $\underline{r}$ where $r \in R_{1} \backslash R$, this follows at once from the previous proposition.

If $R_{1}$ is finite we have a stronger result.

Proposition 2.4. Let $R_{1}$ be a finite set of finitary algebraic relations on $\underline{P}$ and assume that $R_{1}$ yields a duality on $\mathscr{A}$. If $R \subseteq R_{1}$ and $R$ generates $r$ on the test algebra $\underline{r}$ for each $r \in R_{1} \backslash R$, then $R$ yields a duality on $\mathscr{A}$.

Proof. By induction, it suffices to prove the result in the case $\left|R_{1} \backslash R\right|=1$, say $R_{1} \backslash R=\{r\}$. But this follows at once from the previous result.

Proposition 2.5. Assume that $\underline{P}$ has a $(k+1)$-ary near unanimity term $t$ for some $k \geq 2$, that is, $\underline{P}$ satisfies the identities

$$
t(x, \ldots, x, y) \approx t(x, \ldots, x, y, x) \approx \cdots \approx t(y, x, \ldots, x) \approx x .
$$

If $R \subseteq \mathbb{S}\left(\underline{P}^{k}\right)$ generates $r$ on the test algebra $\underline{r}$ for each $r \in \mathbb{S}\left(\underline{P}^{k}\right) \backslash R$, then $R$ yields a duality on $\mathscr{A}$.

Proof. By the $N U$-Duality Theorem (1.18 and 1.19 in [5]), the set $\mathbb{S}\left(\underline{P}^{k}\right)$ yields a duality on $\mathscr{A}$. Now apply the previous proposition.

\section{EXTENDING THE PIGGY BACK PHILOSOPHY}

In [4], a set $R$ of binary algebraic relations on $\underline{P}_{n}$ which yields a natural duality on $\mathbf{B}_{n}$ was found by applying the piggyback technique. In essence, this philosophy says that if a finite algebra $\underline{P}$ has an underlying $\{0,1\}$-distributive lattice structure, we should be able to use the well-understood duality between 
the category $\mathbf{D}$ of bounded distributive lattices and the category $\mathbf{P}$ of compact totally order-disconnected spaces to

(a) help find a structure $\underline{P}=(P ; \mathscr{T}, R)$ which will yield a natural duality on $\mathscr{A}=\mathbb{I} \mathbb{P}(\underline{P})$,

(b) refine the structure $P$ to make it more manageable,

(c) study the structure of the individual relations in $R$,

(d) transfer information between the natural dual category and the restricted D-P dual category thereby enjoying the best of both worlds.

General approaches to (a) have been developed in Davey-Werner [6, 7] and in Davey-Priestley [3]. The companion paper to this, [4], was devoted precisely to (b) and (c) in the case of the varieties $\mathbf{B}_{n}$. The second half of [3] illustrates (d) for certain varieties of Ockham algebras. We now take this philosophy one step further by representing both the natural dual $D(A)=\mathscr{A}\left(A, \underline{P}_{n}\right)$ and the relational structure $P_{n}$ as sets of maps in $\mathbf{P}$ thereby invoking the categorical imperative that everything is a morphism.

We begin with a very brief recap of the restricted D-P duality for $\mathbf{B}_{n}$ given in [10] and the natural duality for $\mathbf{B}_{n}$ established in [4]. Every $\{0,1\}$-distributive lattice $L$ is isomorphic to the lattice of clopen up-sets of its dual space $H(L):=$ $\mathbf{D}(L, 2)$, which is topologized as a subspace of $\underset{\approx}{2}$ and ordered pointwise. As an ordered set, $H(L)$ is isomorphic to the set of prime filters of $L$ ordered by inclusion, and in case $L$ is finite, $H(L)$ has the discrete topology and is order anti-isomorphic to the set of join-irreducible elements of $L$. Moreover, $L$ is pseudocomplemented if and only if $Y=H(L)$ is a p-space, that is, a compact totally order-disconnected space with the property that for each clopen up-set $U$ in $Y$, the set

$$
\downarrow U:=\{z \in Y \mid z \leq y \text { for some } y \in U\}
$$

is clopen. Given $A, B \in \mathbf{B}_{\omega}$, a map $f \in \mathbf{D}(A, B)$ preserves the operation * of pseudocomplementation if and only if $\psi=H(f)$ is a $p$-morphism, that is, it is a continuous order-preserving map with the property that

$$
\psi(\max y)=\max \psi(y) \quad \text { for all } y \in H(B) .
$$

Here $\max z$ denotes the set of maximal points above $z$. The subvariety $\mathbf{B}_{n}$ of $\mathbf{B}_{\omega}$ is characterized by the property that each prime filter of an algebra $A \in \mathbf{B}_{n}$ is contained in at most $n$ maximal filters.

Proposition 3.1. The restriction of the functors $H$ and $K$ establishes a contravariant category equivalence between $\mathbf{B}_{n}$ and $\mathscr{Y}_{n}$, where $\mathscr{Y}_{n}$ is the category whose objects are p-spaces in which each point is majorized by at most $n$ maximal points and whose morphisms are the p-morphisms.

The dual $H\left(\underline{P}_{n}\right)$ of $\underline{P}_{n}$ in $\mathbf{P}$ is the ordered set $V_{n}$ shown in Figure 4. As in [4], we henceforth identify $\underline{P}_{n}$ with the lattice of up-sets of $V_{n}$ : the empty set is denoted by $\perp$, the whole set by $T$ and the co-atom $\{1,2, \ldots, n\}$ by $d$.

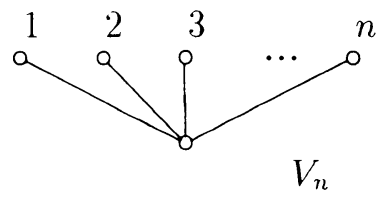

FIGURE 4 
The D-homomorphism $\alpha: \underline{P}_{n} \rightarrow \underset{=}{\mathbf{2}}=\{0,1\}$, which sends $\mathrm{T}$ in $\underline{P}_{n}$ to 1 and all other elements of $\underline{P}_{n}$ to 0, plays a vital role in [4]. Indeed, the general piggyback theory from $[6,7]$ shows that the endomorphisms of $\underline{P}_{n}$ along with the subalgebras of $\underline{P}_{n}^{2}$ which are maximal in

$$
\alpha^{-1}(\leq):=\left\{(a, b) \in \underline{P}_{n}^{2} \mid \alpha(a) \leq a(b)\right\}
$$

yield a duality on $\mathbf{B}_{n}$. Since finite products in $\mathbf{D}$ correspond to disjoint unions in $\mathbf{P}$ and since embeddings in $\mathbf{D}$ correspond to surjections in $\mathbf{P}$, every subalgebra $\underline{r}$ of $\underline{P}_{n}^{2}$ corresponds (in $\mathscr{Y}_{n}$ ) to a pair of $p$-morphisms

$$
\rho_{1}, \rho_{2}: V_{n} \rightarrow Y_{\underline{r}}, \quad \text { where } Y_{\underline{r}}=H(\underline{r}),
$$

which are jointly surjective (that is, for all $y \in Y_{\underline{r}}$ there exists $i \in V_{n}$ such that either $\rho_{1}(i)=y$ or $\left.\rho_{2}(i)=y\right)$. Furthermore, $r \subseteq \alpha^{-1}(\leq)$ if and only if $\rho_{1}(0) \leq \rho_{2}(0)$, and $r$ will be maximal in $\alpha^{-1}(\leq)$ if and only if $\rho_{1}(0)<\rho_{2}(0)$ and $Y_{\underline{r}}$ has exactly $n$ maximal elements; then $\left|Y_{\underline{r}}\right|=n+2$ and $Y_{\underline{r}}$ has the shape shown in Figure 5 for some $l$ with $1 \leq l \leq n$. Since, for fixed $n$, the ordered set in Figure 5 depends only upon the integer $l$, we denote it by $Y_{l}$. It is often helpful to think of $\rho_{1}$ and $\rho_{2}$ as providing new labellings of the maximals of $Y_{l}$ : if $\rho_{1}(i)=j$, then label $j$ with the symbol $i$, and if $\rho_{2}(i)=j$ then label $j$ with the symbol $\tilde{i}$.

Every partition $p=\left(k_{1}, k_{2}, \ldots, k_{l}\right)$ of the integer $n$ with $k_{1} \geq k_{2} \geq \cdots \geq k_{l}$ gives rise to a (left-packed) subalgebra $M\left(k_{1}, k_{2}, \ldots, k_{l}\right)$ of $\underline{P}_{n}^{2}$ corresponding to the pair $\rho_{1}, \rho_{2}: V_{n} \rightarrow Y_{l}$ defined as follows:

$$
\begin{gathered}
\rho_{1}(i)=i \quad \text { for all } i \in V_{n}=\{0,1, \ldots, n\} \\
\rho_{2}(0)=\tilde{0} \quad \text { and } \quad \rho_{2}(i)=j \quad \text { for all } i \in X_{j}
\end{gathered}
$$

where

$$
\begin{aligned}
X_{1} & =\left\{1, \ldots, k_{1}\right\} \\
X_{2} & =\left\{k_{1}+1, \ldots, k_{1}+k_{2}\right\} \\
X_{3} & =\left\{k_{1}+k_{2}+1, \ldots, k_{1}+k_{2}+k_{3}\right\}, \ldots, \\
X_{l} & =\left\{n-k_{l}+1, \ldots, n\right\}
\end{aligned}
$$

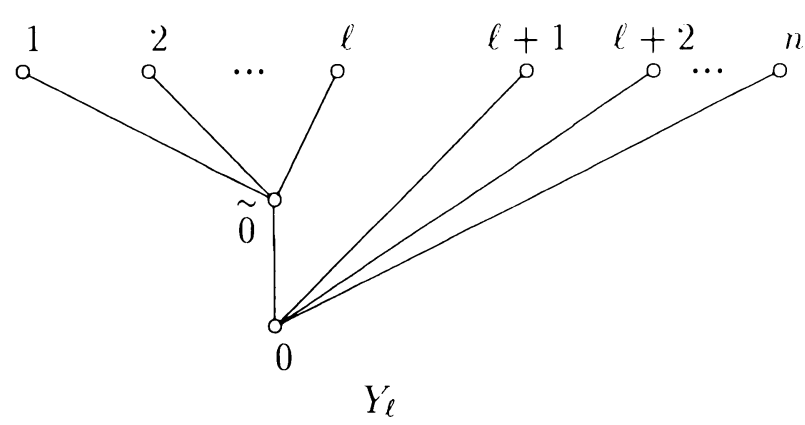

FIGURE 5 


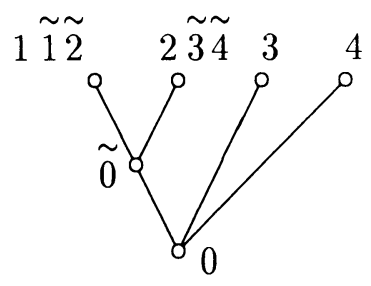

$Y_{(2,2)}$

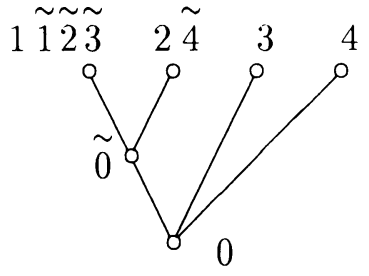

$Y_{(3,1)}$

FigURE 6

To illustrate the notation, consider the relations $r^{\prime}$ and $r^{\prime \prime}$ associated with the partitions $(2,2)$ and $(3.1)$ of $n=4$. The corresponding labelings of $Y_{2}$ are shown in Figure 6. The jointly surjective maps $\rho_{1}, \rho_{2}$ are given by

$$
\begin{aligned}
\rho_{1} & =\text { id } \quad \text { for both } r^{\prime} \text { and } r^{\prime \prime}, & & \\
\rho_{2}(1) & =\rho_{2}(2)=1, \quad \rho_{2}(3)=\rho_{2}(4)=2 & & \text { for } r^{\prime}, \\
\rho_{2}(1) & =\rho_{2}(2)=\rho_{2}(3)=1, \quad \rho_{2}(4)=2 & & \text { for } r^{\prime \prime} .
\end{aligned}
$$

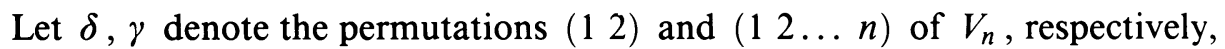
and let $f_{\delta}$ and $f_{\gamma}$ be the corresponding automorphisms of $\underline{P}_{n}$. Let $f_{\nu}$ be the endomorphism of $\underline{P}_{n}$ corresponding to the constant map $\nu: V_{n} \rightarrow V_{n}$ onto $\{1\}$. The following is the main result of [4] and shows that there is a natural duality for $\mathbf{B}_{n}$ requiring at most $p(n)+3$ binary algebraic relations.

Proposition 3.2. Let $n \geq 1$. Then $S \cup G$ yields a duality on $\mathbf{B}_{n}$, where

(i) $S=\left\{\boldsymbol{M}\left(k_{1}, k_{2}, \ldots, k_{l}\right) \mid\left(k_{1}, k_{2}, \ldots, k_{l}\right)\right.$ is a partition of $\left.n\right\}$,

(ii) $G$ is (the set of graphs of) a generating set for the endomorphism monoid of $\underline{P}_{n}$;

$$
G= \begin{cases}\left\{f_{\nu}\right\} & \text { if } n=1, \\ \left\{f_{\nu}, f_{\delta}\right\} & \text { if } n=2, \\ \left\{f_{\nu}, f_{\delta}, f_{\gamma}\right\} & \text { if } n \geq 3,\end{cases}
$$

suffices, where $f_{\nu}, f_{\delta}$, and $f_{\gamma}$ are defined as above.

In the next section, a seminal role will be played by the dual in $\mathscr{Y}_{n}$ of the free algebra $F \mathbf{B}_{n}(1)$. Since $\mathbf{B}_{1}$ is characterized by the Stone identity, $x^{* *} \vee x^{*} \approx 1$, it is easily seen that $F \mathbf{B}_{1}(1)$ is isomorphic (as a lattice) to the direct product of a two-element and a three-element chain; at the other end of the varietal spectrum, $F \mathbf{B}_{\omega}(1)$ is as shown in Figure 7 (see [8]). Since $F \mathbf{B}_{\omega}(1)$ belongs to $\mathbf{B}_{2}$ it follows that $F \mathbf{B}_{n}(1) \cong F \mathbf{B}_{\omega}(1)$ for all $n \geq 2$.

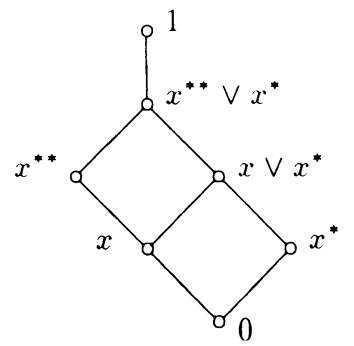

$F \mathbf{B}_{n}(1)$ for $n \geqslant 2$

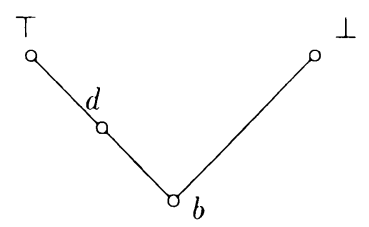

$F=H\left(F \mathbf{B}_{n}(1)\right)$ for $n \geqslant 2$

FIGURE 7 
Let $F:=H\left(F \mathbf{B}_{n}(1)\right)$, as shown in Figure 7. Then we have natural bijections

$$
P_{n} \leftrightarrow \mathbf{B}_{n}\left(F \mathbf{B}_{n}(1), \underline{P}_{n}\right) \leftrightarrow \mathscr{Y}_{n}\left(H\left(\underline{P}_{n}\right), H\left(F \mathbf{B}_{n}(1)\right)\right)=\mathscr{Y}_{n}\left(V_{n}, F\right) .
$$

For $2 \leq n<\omega$, the composite bijection $\Gamma: P_{n} \rightarrow \mathscr{Y}_{n}\left(V_{n}, F\right)$ is defined as follows: $\Gamma(a)=\psi_{a}: V_{n} \rightarrow F$ for all $a \in P_{n}$, where $\psi_{a}$ is the unique $p$ morphism from $V_{n}$ to $F$ satisfying $\psi_{a}(0)=a$ when $a \in\{\perp, d, T\}$, namely,

$$
\begin{aligned}
& \psi_{\top}(i)=\top \quad \text { for all } i \in V_{n}, \\
& \psi_{\perp}(i)=\top \quad \text { for all } i \in V_{n}, \\
& \psi_{d}(i)= \begin{cases}\top & \text { if } i \in\{1,2, \ldots, n\}, \\
d & \text { if } i=0,\end{cases}
\end{aligned}
$$

and for $\varnothing \subset a \subset d$, the map $\psi_{a}: V_{n} \rightarrow F$ is the $p$-morphism which on the maximals of $V_{n}$ is the characteristic function of a, namely,

$$
\psi_{a}(i)= \begin{cases}\top & \text { if } i \in a, \\ \perp & \text { if } i \in d \backslash a, \\ b & \text { if } i=0 .\end{cases}
$$

The notation for the elements of $F$ given in Figure 7 was chosen to make the definition of $\Gamma$ natural (the letter $b$ should remind the reader that $p$ morphisms $\psi: V_{n} \rightarrow F$ with $\psi(0)=b$ correspond to the proper, nontrivial Boolean elements of $\underline{P}_{n}$ ).

Let $A \in \mathbf{B}_{n}$ and denote $H(A)$ by $Y_{A}$. Then the functor $H: \mathbf{B}_{n} \rightarrow \mathscr{Y}_{n}$ gives a bijection

$$
D(A):=\mathbf{B}_{n}\left(A, \underline{P}_{n}\right) \stackrel{H}{\longrightarrow} \mathscr{Y}_{n}\left(H\left(\underline{P}_{n}\right), H(A)\right)=\mathscr{Y}_{n}\left(V_{n}, Y_{A}\right) .
$$

Our next result, whose proof we leave to the reader, shows that this bijection carries over the relational structure in a very natural way.

Proposition 3.3. Assume that $\underline{r} \leq \underline{P}_{n}^{2}$ and that $\underline{r}$ corresponds to the pair of jointly surjective p-morphisms $\rho_{1}, \rho_{2}: V_{n} \rightarrow Y_{l}$. Interpret the relation $r$ on the set $\mathscr{Y}_{n}\left(V_{n}, Y_{A}\right)$ by declaring that a pair of maps $\varphi_{1}, \varphi_{2} \in \mathscr{Y}_{n}\left(V_{n}, Y_{A}\right)$ satisfy $\left(\varphi_{1}, \varphi_{2}\right) \in r$ if and only if $\left(\varphi_{1}, \varphi_{2}\right)$ factors in $\mathscr{Y}_{n}$ through $\left(\rho_{1}, \rho_{2}\right)$, that is, there exists $\mu \in \mathscr{Y}_{n}\left(Y_{l}, Y_{A}\right)$ such that the diagram below commutes.

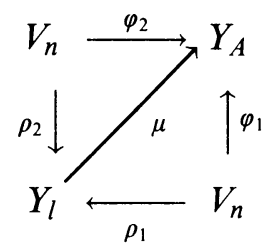

(i) The natural bijection between $D(A)=\mathbf{B}_{n}\left(A, \underline{P}_{n}\right)$ and $\mathscr{Y}_{n}\left(V_{n}, Y_{A}\right)$ given by the functor $H$ is an isomorphism with respect to the relation $r$.

(ii) Every $p$-morphism $\mu: Y_{l} \rightarrow Y_{A}$ gives rise to a pair of maps $\varphi_{1}, \varphi_{2} \in$ $\mathscr{Y}_{n}\left(V_{n}, Y_{A}\right)$ with $\left(\varphi_{1}, \varphi_{2}\right) \in r$, namely $\varphi_{1}:=\mu \circ \rho_{1}$ and $\varphi_{2}:=\mu \circ \rho_{2}$.

The functor $H: \mathbf{B}_{n} \rightarrow \mathscr{Y}_{n}$ also gives a monoid isomorphism

$$
\text { End } \underline{P}_{n}=\mathbf{B}_{n}\left(\underline{P}_{n}, \underline{P}_{n}\right) \stackrel{H}{\longrightarrow} \mathscr{Y}_{n}\left(V_{n}, V_{n}\right)=\text { End } V_{n} \text {. }
$$


Of course, each element $e \in$ End $V_{n}$ acts on $\mathscr{Y}_{n}\left(V_{n}, Y_{A}\right)$ by composition

$$
\left(\forall \psi \in \mathscr{Y}_{n}\left(V_{n}, Y_{A}\right)\right) e(\psi):=\psi \circ e \in \mathscr{Y}_{n}\left(V_{n}, Y_{A}\right) .
$$

Thus we may take any family $S$ of binary algebraic relations on $\underline{P}_{n}$ and any set $G$ of endomorphisms of $\underline{P}_{n}$ and interpret them on $\mathscr{Y}_{n}\left(V_{n}, Y_{A}\right)$ in such a way that $\left(\mathscr{Y}_{n}\left(V_{n}, Y_{A}\right) ; G, S\right)$ is an isomorphism copy of $\left(\mathbf{B}_{n}\left(A, \underline{P}_{n}\right) ; G, S\right)$. In particular, since $P_{n}$ is isomorphic to $D\left(F \mathbf{B}_{\omega}(1)\right)$, it follows that the structure $\left(\mathscr{Y}_{n}\left(V_{n}, F\right) ; \mathscr{T}, G, S\right)$ is an isomorphic copy of $P_{n}=\left(P_{n} ; \mathscr{T}, G, S\right)$. This turns out to be a particularly useful view of the world as will be seen in the following two sections.

\section{UNAVOIDABLE RELATIONS}

The main result of [4] (Proposition 3.2 above) tells us that the left-packed (partition-induced) relations along with a generating set for End $\underline{P}_{n}$ yield a duality on $\mathbf{B}_{n}$. Our first unavoidability result shows that the endomorphism monoid plays an essential role in such a duality.

Theorem 4.1. Let $S$ be a family of left-packed subalgebras of $\underline{P}_{n}^{2}$ and let $G$ be a family of endomorphisms of $\underline{P}_{n}$. If $\underline{P}_{n}=\left(P_{n} ; \mathscr{T}, G, S\right)$ yields a duality on $\mathbf{B}_{n}$, then $G$ generates End $\underline{P}_{n}$ as a monoid.

Proof. Assume that $P_{n}=\left(P_{n} ; \mathscr{T}, G, S\right)$ yields a duality on $\mathbf{B}_{n}$. We shall show that $G$ must contain at least one endomorphism which is not an automorphism along with a generating set for Aut $\underline{P}_{n}$. Note that, regarded as a subalgebra of $\underline{P}_{n}^{2}$, each endomorphisms of $\underline{P}_{n}$ is isomorphic to $\underline{P}_{n}$; whence the appropriate test algebra for an endomorphism is $\underline{P}_{n}$ itself.

Let $\psi: D\left(\underline{P}_{n}\right)=$ End $\underline{P}_{n} \rightarrow P_{n}$ be the constant map onto $\{d\}$. Since the automorphisms of $\underline{P}_{n}$ all map $d$ to $d$ and since every left-packed subalgebra of $\underline{P}_{n}^{2}$ contains the pair $(d, d)$, the map $\psi$ preserves all automorphisms of $\underline{P}_{n}$ and all left-packed subalgebras of $\underline{P}_{n}^{2}$. Clearly, $\psi$ does not preserve any endomorphism which is not an automorphism since such endomorphisms map $d$ to $\mathrm{T}$. It follows that the set $G$ contains at least one endomorphism which is not an automorphism.

Since $\underline{P}_{1}$ has no nonidentity automorphisms, we may now assume that $n \geq$ 2. Let $H$ be the subgroup of Aut $\underline{P}_{n}$ generated by the automorphisms in $G$. Define a map $\varphi: D\left(\underline{P}_{n}\right)=$ End $\underline{P}_{n} \rightarrow P_{n}$ by

$$
\varphi(e)= \begin{cases}T & \text { if } e \in \operatorname{End} \underline{P}_{n} \backslash \text { Aut } \underline{P}_{n}, \\ d & \text { if } e \in H, \\ T & \text { if } e \in \text { Aut } \underline{P}_{n} \backslash H .\end{cases}
$$

We claim that $\varphi$ preserves all left-packed subalgebras of $\underline{P}_{n}^{2}$ and preserves an endomorphism if and only if it is in either End $\underline{P}_{n} \backslash$ Aut $\underline{P}_{n}$ or in $H$. We conclude at once that $H=$ Aut $\underline{P}_{n}$.

Let $\underline{r}$ be a left-packed subalgebra of $\underline{P}_{n}^{2}$ and suppose, by way of contradiction, that $e, f \in$ End $\underline{P}_{n}$ with $(e, f) \in r$ but $(\varphi(e), \varphi(f)) \notin r$. Since every left-packed subalgebra of $\underline{P}_{n}^{2}$ contains $(d, d),(d, \mathrm{~T})$, and $(\mathrm{T}, \mathrm{T})$, it follows that $(\varphi(e), \varphi(f))=(T, d)$, whence $f \in H$ and $e \notin H$. Since $(e, f) \in r$, we have

$$
\left\{(e(a), f(a)) \mid a \in P_{n}\right\} \subseteq r .
$$


As $f$ is surjective, it follows that the second projection restricted to $r$ maps onto $P_{n}$. The only left-packed relation with this property is the order corresponding to the partition $(1,1, \ldots, 1)$. Thus,

$$
r=\left\{(x, x) \mid x \in P_{n}\right\} \cup\{(d, \top)\} \text {. }
$$

Hence $e(a)=f(a)$ for all $a \in P_{n} \backslash\{d\}$ and consequently (as $n \geq 2$ ) we have $e=f$, contradicting the fact that $f \in H$ and $e \notin H$. Thus $\varphi$ preserves all left-packed relations.

Let $f \in$ End $\underline{P}_{n} \backslash$ Aut $\underline{P}_{n}$ and let $e \in$ End $\underline{P}_{n}$. Then

$$
\varphi(f(e))=\varphi(e \circ f)=\top \quad \text { as } e \circ f \in \text { End } \underline{P}_{n} \backslash \text { Aut } \underline{P}_{n},
$$

and

$$
f(\varphi(e)) \in f(\{d, \top\})=\{\top\} \quad \text { as } f \in \text { End } \underline{P}_{n} \backslash \text { Aut } \underline{P}_{n},
$$

and therefore $\varphi$ preserves $f$.

Now assume that $f \in H$ and let $e \in$ End $\underline{P}_{n}$. Using the fact that $e \circ f \in H$ if and only if $e \in H$, it is easily seen that

$$
\varphi(f(e))=\varphi(e \circ f)= \begin{cases}d & \text { if } e \in H \\ \top & \text { if } e \in \text { End } \underline{P}_{n} \backslash H\end{cases}
$$

Since $f$ is an automorphism, we find

$$
f(\varphi(e))= \begin{cases}f(d)=d & \text { if } e \in H, \\ f(T)=\top & \text { if } e \in \text { End } \underline{P}_{n} \backslash H .\end{cases}
$$

Hence $\varphi$ preserves $f$.

Finally, let $f \in$ Aut $\underline{P}_{n} \backslash H$. Then $\varphi(f($ id $))=\varphi($ id $\circ f)=\varphi(f)=$ T, while $f(\varphi(\mathrm{id}))=f(d)=d$, whence $\varphi$ does not preserve $f$.

We turn now to the binary algebraic relations on $\underline{P}_{n}$ which are not (graphs of) endomorphisms. Let $r$ be a binary algebraic relation on $\underline{P}_{n}$. We shall refer to the ordered set $Y_{r}=H(\underline{r})$ (up to order-isomorphism) as the shape of $r$. thus two algebraic binary relations have the same shape if and only if they are isomorphic as algebras. If $\underline{r}$ is a subalgebra of $\underline{P}_{n}^{2}$ which is maximal in $\alpha^{-1}(\leq)$, in particular if $\underline{r}=M\left(k_{1}, \ldots, k_{l}\right)$ for some partition $\left(k_{1}, k_{2}, \ldots, k_{l}\right)$ of $n$, then the shape of $r$ is uniquely determined by the number, $l$, of maximals above $\tilde{0}$ (see Figure 5); in which case, we refer to $r$ as a relation of shape $l$. Conversely, if $r$ is a relation of shape $Y_{l}$, then either $\underline{r}$ or its converse, $\underline{r}^{\smile}:=\{(a, b) \mid(b, a) \in r\}$, is maximal in $\alpha^{-1}(\leq)$. Since the shape of a relation is determined only up to isomorphism, the concept blurs the distinction between a relation and its converse. This is deliberate as it is a completely trivial change in the character of $P_{n}$ to replace one of the relations by its converse.

Lemma 4.2. Let $\underline{r}$ and $\underline{s}$ be subalgebras of $\underline{P}_{n}^{2}$ which are maximal in $\alpha^{-1}(\leq)$. Then $s$ has the same shape as $r$ if and only if there is a surjective p-morphism from $Y_{\underline{s}}$ onto $Y_{\underline{r}}$.

Proof. Let $\mu: Y_{\underline{s}} \rightarrow Y_{\underline{r}}$ be a surjective $p$-morphism with $\underline{r}$ and $\underline{s}$ maximal in $\alpha^{-1}(\leq)$. Since $\left|Y_{\underline{s}}\right|=\left|Y_{\underline{r}}\right|=n+2$, it follows that the most $\mu$ can do is shuffle maximal elements. In order to satisfy $\mu(\max \tilde{0})=\max \mu(\tilde{0})$, we must have $|\max \tilde{0}|=|\max \mu(\tilde{0})|$, whence $\mu$ is a $p$-isomorphism. 
Proposition 4.3. Let $\underline{r}$ be a subalgebra of $\underline{P}_{n}^{2}$ which is maximal in $\alpha^{-1}(\leq)$. Then there is a map $\Phi: \mathbf{B}_{n}\left(\underline{r}, \underline{P}_{n}\right) \rightarrow P_{n}$ which (a) preserves the action of every endomorphism of $\underline{P}_{n}$, and (b) preserves a binary algebraic relation $s$ if and only if the shape of $s$ is different from the shape of $r$.

Proof. We shall use the approach developed in the previous section and replace $\mathbf{B}_{n}\left(\underline{r}, \underline{P}_{n}\right)$ by $\mathscr{Y}_{n}\left(V_{n}, Y_{r}\right)$ and $P_{n}$ by $\mathscr{Y}_{n}\left(V_{n}, F\right)$. Since the case $n=1$ is a simple modification of the argument below and requires a different $F$ (see Figure 7), we shall assume that $n \geq 2$.

Let $\underline{r} \leq \underline{P}_{n}^{2}$ be maximal in $\alpha^{-1}(\leq)$. We require a map $\Phi: \mathscr{Y}_{n}\left(V_{n}, Y_{\underline{r}}\right) \rightarrow$ $\mathscr{Y}_{n}\left(V_{n}, F\right)$ which

(i) preserves the action of each $e \in \mathscr{Y}_{n}\left(V_{n}, V_{n}\right)$,

(ii) does not preserve relations $s$ with $Y_{\underline{s}} \cong Y_{\underline{r}}$, and

(iii) preserves relations $s$ with $Y_{\underline{s}} \neq Y_{\underline{r}}$.

Consider the map $\beta: Y_{\underline{r}} \rightarrow F$ defined as

$$
\beta(y)= \begin{cases}\top & \text { if } y \text { is maximal in } Y_{\underline{r}}, \\ d & \text { if } y=\tilde{0}, \\ \top & \text { if } y=0 .\end{cases}
$$

Although $\beta$ is not even order-preserving, we claim that $\beta \circ \psi \in \mathscr{Y}_{n}\left(V_{n}, F\right)$ for all $\psi \in \mathscr{Y}_{n}\left(V_{n}, Y_{\underline{r}}\right)$. Firstly, $\beta \circ \psi$ is order-preserving since $\{0, \tilde{0}\} \nsubseteq \psi\left(V_{n}\right)$ and $0 \leq \tilde{0}$ is the only order relation on $Y_{\underline{r}}$ which $\beta$ fails to preserve. Since $\beta$ satisfies

$$
\left(\forall y \in Y_{\underline{r}}\right) \quad \beta(\max y)=\max \beta(y),
$$

it now follows that $\beta \circ \psi \in \mathscr{Y}_{n}\left(V_{n}, F\right)$. Hence $\Phi: \mathscr{Y}_{n}\left(V_{n}, Y_{\underline{r}}\right) \rightarrow \mathscr{Y}_{n}\left(V_{n}, F\right)$, given by $\psi \mapsto \beta \circ \psi$, is well defined.

It is trivial that $\Phi$ preserves the action of $\mathscr{Y}_{n}\left(V_{n}, V_{n}\right)$ since this is just the associative law for composition of maps:

$$
\Phi(e(\psi))=\beta \circ(\psi \circ e)=(\beta \circ \psi) \circ e=e(\Phi(\psi)) .
$$

Thus (i) holds.

Now consider (ii). Let $s$ be a binary algebraic relation on $\underline{P}_{n}$ which has the same shape as $r$ and let $\sigma_{1}, \sigma_{2}: V_{n} \rightarrow Y_{s}$ be the jointly surjective maps which correspond to $s$. Hence there is a $p$-isomorphism $\mu: Y_{\underline{s}} \rightarrow Y_{\underline{r}}$. Thus $\psi_{1}:=\mu \circ \sigma_{1}$ and $\psi_{2}:=\mu \circ \sigma_{2}$ are elements of $\mathscr{Y}_{n}\left(V_{n}, Y_{\underline{r}}\right)$ with $\left(\psi_{1}, \psi_{2}\right) \in s$ (by Proposition 3.3(ii)). Note that for all $Y \in \mathscr{Y}_{n}$, if $\varphi_{1}, \varphi_{2} \in \mathscr{Y}_{n}\left(V_{n}, Y\right)$ with $\left(\varphi_{1}, \varphi_{2}\right) \in s$, then there exists $\eta: Y_{\underline{s}} \rightarrow Y$ with $\varphi_{1}=\eta \circ \sigma_{1}$ and $\varphi_{2}=\eta \circ \sigma_{2}$, and hence

$$
\varphi_{1}(0)=\eta\left(\sigma_{1}(0)\right)=\eta(0) \leq \eta(\tilde{0})=\eta\left(\sigma_{2}(0)\right)=\varphi_{2}(0) .
$$

Thus $\left(\beta \circ \psi_{1}, \beta \circ \psi_{2}\right) \notin s$ on $\mathscr{Y}_{n}\left(V_{n}, F\right)$ since

$$
\begin{aligned}
\left(\beta \circ \psi_{1}\right)(0) & =\beta\left(\mu\left(\sigma_{1}(0)\right)\right)=\beta(\mu(0))=\beta(0)=\top \\
& \not \leq d=\beta(\tilde{0})=\beta(\mu(\tilde{0}))=\beta\left(\mu\left(\sigma_{2}(0)\right)\right)=\left(\beta \circ \psi_{1}\right)(0) .
\end{aligned}
$$

Consequently, $\Phi$ does not preserve $s$, whence (ii) holds.

Finally, consider (iii). Assume that $s$ does not have the same shape as $r$ and let $\sigma_{1}, \sigma_{2}: V_{n} \rightarrow Y_{\underline{s}}$ be the jointly surjective maps corresponding to $s$. Clearly, $Y_{\underline{S}}$ has at most two nonmaximal elements $\left(0:=\sigma_{1}(0)\right.$ and $0:=\sigma_{2}(0)$, 


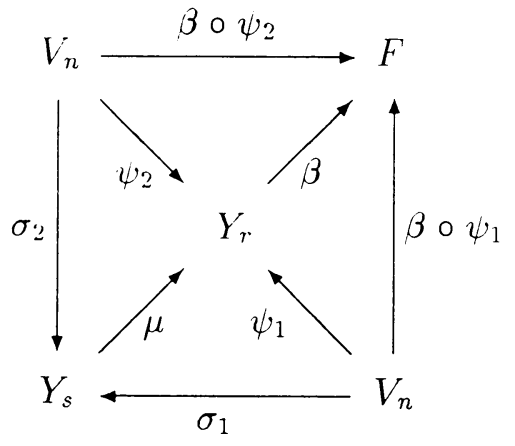

FIGURE 8

which may coincide). Of course, $\Phi$ preserves $s$ if and only if it preserves the converse relation $s^{\smile}$. Since $\left(\sigma_{2}, \sigma_{1}\right)$ is the pair of $p$-morphisms corresponding to $s^{\smile}$, without loss of generality we have only three cases: (a) $0=\tilde{0}$, (b) $0 \| \tilde{0}$, (c) $0<\tilde{0}$.

Assume that $\psi_{1}, \psi_{2} \in \mathscr{Y}_{n}\left(V_{n}, Y_{r}\right)$ with $\left(\psi_{1}, \psi_{2}\right) \in s$. Then there exists $\mu: Y_{s} \rightarrow Y_{r}$ such that the diagram in Figure 8 commutes. To see that $\left(\beta \circ \psi_{1}, \bar{\beta} \circ \psi_{2}\right) \in s$ it suffices to show that $\beta \circ \mu: Y_{\underline{s}} \rightarrow F$ is a $p$-morphism

If $\mu$ is not surjective, then $\beta \circ \mu$ is easily seen to be a $p$-morphism (see the proof above that $\psi$ is well defined); this covers Case (a). Now assume that $\mu$ is surjective; consequently, $\mu(\{0, \tilde{0}\})=\{0, \tilde{0}\}$. Case (b) presents no problems: since $0 \| \tilde{0}$ in $Y_{\underline{s}}$, the fact that $\beta$ does not preserve the relation $0 \leq \tilde{0}$ in $Y_{\underline{r}}$ is of no consequence. We are reduced to Case (c). As $0<\tilde{0}$ in $Y_{s}$, we have $\left|Y_{\underline{s}}\right| \leq n+2$, and since $\mu$ is surjective we conclude that $\left|Y_{\underline{s}}\right|=n+2$. Hence $\underline{s}$ is maximal in $\alpha^{-1}(\leq)$ and Lemma 4.2 shows that $s$ has the same shape as $r$. This contradiction concludes the proof.

Since the map $\Phi$ preserves the action of an endomorphism $e$ of $\underline{P}_{n}$ on a dual space $D(A)=\mathbf{B}_{n}\left(A, \underline{P}_{n}\right)$ if and only if it preserves the graph of $e$, and since such a graph is never maximal in $\alpha^{-1}(\leq)$, there is a degree of redundancy in both the statement of Proposition 4.3 and its proof.

It is of interest to write down the map $\Phi$ explicitly as a map into $P_{n}$ via the bijection $\Gamma$ between $P_{n}$ and $\mathscr{Y}_{n}\left(V_{n}, F\right)$. Since $\beta$ maps $Y_{r}$ onto $\{d, T\} \subseteq F$, it follows that $\Phi$ maps $\mathscr{Y}_{n}\left(V_{n}, Y_{r}\right)$ onto $\{d, T\} \subseteq P_{n}$ (consult the definition of $\Gamma$ ). Indeed, for all $\psi \in \mathscr{Y}_{n}\left(V_{n}, Y_{\underline{r}}\right)$, we have $\Phi(\psi) \in P_{n}$ defined by

$$
\Phi(\psi):= \begin{cases}T & \text { if } \psi(0) \text { is maximal in } Y_{\underline{r}}, \\ d & \text { if } \psi(0)=\tilde{0}, \\ \top & \text { if } \psi(0)=0 .\end{cases}
$$

Our first minimality result is an easy consequence of Proposition 4.3.

Theorem 4.4. Let $S$ be a family of binary algebraic relations on $\underline{P}_{n}$ such that $P_{n}:=\left(P_{n} ; \mathscr{T}, G, S\right)$ yields a duality on $\mathbf{B}_{n}$ for some set $G$ of endomorphisms of $\underline{P}_{n}$. Then for all $l$ with $1 \leq l \leq n$, the set $S$ includes at least one relation $r$ of shape $l$. Hence $|S| \geq n$.

Proof. Let $\underline{r}$ be a subalgebra of $\underline{P}_{n}^{2}$ which is maximal in $\alpha^{-1}(\leq)$ and suppose that $S$ includes no relation with the same shape as $r$. By Proposition 
4.3, there exists a map $\Phi$ in the second dual of $\underline{r}, \Phi: \mathbf{B}_{n}\left(\underline{r}, \underline{P}_{n}\right) \rightarrow \underline{P}_{n}$, which does not preserve the relation $r$. But for all $a \in r$ the evaluation map $e_{\underline{r}}(a): \mathbf{B}_{n}\left(\underline{r}, \underline{P}_{n}\right) \rightarrow \underline{P}_{n}$ given by $f \mapsto f(a)$ preserves every subalgebra of $\underline{P}_{n}^{2}$. Consequently,

$$
e_{\underline{r}}: \underline{r} \rightarrow \mathscr{X}\left(\mathbf{B}_{n}\left(\underline{r}, \underline{P}_{n}\right), \underline{P}_{n}\right)=E D(\underline{r})
$$

is not an isomorphism, contradicting the fact that $P_{n}$ yields a duality. Hence $S$ includes at least one relation which as the same shape as $\underline{r}$. Since for each $n$ there are exactly $n$ possible shapes for the subalgebras of $\underline{P}_{n}^{2}$ which are maximal in $\alpha^{-1}(\leq)$, we conclude that $|S| \geq n$.

This theorem leads us to suggest the study of unavoidable relations. Since this is the first result of its kind, it is unclear what the most appropriate definition will be. What follows is a first pass which, at least, is suitable for the particular context of this paper. For any set $S$ of binary relations define $S^{\smile}:=\left\{s^{\smile} \mid s \in\right.$ $S$ \}. A set $S$ of binary algebraic relations on $\underline{P}$ is unavoidable (among binary relations) if any set $R$ of binary algebraic relations which yields a duality on $\mathscr{A}=\operatorname{ISP}(\underline{P})$ intersects $S \cup S^{\smile}$. A relation $s$ is called unavoidable if $\{s\}$ is . Thus Theorem 4.4 tells us that, for each $l$ with $1 \leq l \leq n$, the set of binary algebraic relations of shape $l$ is unavoidable.

For $1 \leq n \leq 3$, we have the strongest possible minimality result. The relations $\leq, \dashv$, and $\triangleleft$ were defined in the introduction.

Theorem 4.5. Let $n \in\{1,2,3\}$ and let $G$ be the generating set for the endomorphism monoid of $\underline{P}_{n}$ described in Proposition 3.2. Let $S_{n} \subseteq \mathbb{S}\left(\underline{P}_{n}^{2}\right)$ be given by $S_{1}=\{\leq\}, S_{2}=\{\leq,-\}$, and $S_{3}=\{\leq,-1, \triangleleft\}$. Then, up to replacing $a$ relation by its converse, $S_{n}$ is the smallest set of binary algebraic relations such that $P_{n}=\left(P_{n} ; \mathscr{T}, G, S_{n}\right)$ yields a duality on $\mathbf{B}_{n}$.

Proof. The result follows from Proposition 3.2 (which says that $P_{n}$ yields a duality on $\left.\mathbf{B}_{n}\right)$, and Theorem 4.4 which implies that if $P_{n}^{\prime}:=\left(P_{n} ; G, S, \mathscr{T}\right)$ yields a duality on $\mathbf{B}_{n}$ then, up to replacing a relation by its converse, we have $S_{n} \subseteq S$. The only additional observation required is that if $1 \leq l \leq n \leq 3$, then there are precisely two binary algebraic relations on $\underline{P}_{n}$ of shape $l$ each being the converse of the other.

Thus for $n \in\{1,2,3\}$, each of the relations listed above in $S_{n}$ is unavoidable.

\section{Optimal dualities}

The final result of the previous section shows that the dualities given in $[1,2$, $4,6,7]$ for $\mathbf{B}_{1}, \mathbf{B}_{2}$, and $\mathbf{B}_{3}$ are optimal in a very strong sense. For $n \geq 4$, it would be unreasonable to hope for such a sharp result: indeed, two partitions of $n$ of the same length, say $\left(k_{1}, \ldots, k_{l}\right)$ and $\left(k_{1}^{\prime}, \ldots, k_{l}^{\prime}\right)$ can lead to radically different left-packed relations (see Propositions 4.1 and 4.2 in [4]) although they are both of shape $l$. While Theorem 4.4 says that at least one relation of shape $l$ must be chosen, there is no obvious reason to choose one of these relations over the other.

Our aim in this section is to establish an optimality result appropriate to a duality for $\mathbf{B}_{n}$ when $n \geq 4$. We prove that it is possible to obtain the lower bound of $n$ (given by Theorem 4.4) on the size of a set $S$ of binary algebraic relations on $\underline{P}_{n}$ such that $\underline{P}_{n}:=\left(P_{n} ; \mathscr{T}, G, S\right)$ yields a duality on $\mathbf{B}_{n}$ : simply 
include in $S$ exactly one (left-packed, partition-induced) relation of each shape $l$.

Theorem 5.1. Let $n \geq 4$. Let $G=\left\{f_{\nu}, f_{\delta}, f_{\gamma}\right\}$, as defined in $\S 3$, and let $S$ be any family of binary algebraic relations on $\underline{P}_{n}$ such that $\underline{P}_{n}:=\left(P_{n} ; \mathscr{T}, G, S\right)$ yields a duality on $\mathbf{B}_{n}$. Then $S$ contains at least one relation $r$ of shape $l$ for $1 \leq l \leq n$. If $S=\left\{r_{1}, \ldots, r_{n}\right\}$, where $r_{l}$ is any binary algebraic relation on $\underline{P}_{n}$ of shape $l$, then $\underline{P}_{n}$ yields a duality on $\mathbf{B}_{n}$.

Our strategy for proving the optimal-duality theorem will be as follows. We combine Proposition 2.4, on the use of test algebras, with an inductive argument whereby we obtain, successively for $m=2,3, \ldots, n$, a duality-generating set of relations containing just one (left-packed) relation of shape $k$ for $1 \leq k \leq$ $m$ and all binary algebraic relations of shape $k$ for $m<k \leq n$. This is accomplished by showing that for any shape $l$ with $1<l<n$, a single leftpacked relation of shape $l$ together with all relations of higher shape suffice to generate all left-packed relations of shape $l$ (Lemma 5.6). The proof of Lemma 5.6 depends on a sequence of preliminary lemmas (5.2-5.5). We first show that if $1<l<n$, then a $p$-morphism into $Y_{l}$ is determined by the restriction of its domain and codomain to maximals. This is then used in Lemma 5.3 to show that if $1<l<n$, then every relation which is maximal in $\alpha^{-1}(\leq)$ is the graph of a partial map on $\mathscr{Y}_{n}\left(V_{n}, Y_{l}\right)$. Lemmas 5.4 and 5.5 say, in essence, that if $r$ and $s$ are left-packed relations of the same shape, $l$, then there is a relation $t$ of higher shape such that the corresponding partial maps on $\mathscr{Y}_{n}\left(V_{n}, Y_{l}\right)$ satisfy $t \circ r=s$.

Throughout the remainder of this section we shall assume that $n \geq 4$.

If $X$ and $Y$ are $p$-spaces and $\varphi: X \rightarrow Y$ is a $p$-morphism, then $\varphi$ maps the maximals of $X$ into the maximals of $Y$. The map $\hat{\varphi}$ obtained by restricting both the domain and codomain of $\varphi$ to the maximals will be referred to as the top of $\varphi$.

Lemma 5.2. Let $1<l<n$. Then for each $p$-space $Y$, every $p$-morphism $\varphi: Y \rightarrow Y_{l}$ is uniquely determined by its top, $\hat{\varphi}$.

Proof. Let $y \in Y$. There are only three possibilities for $\hat{\varphi}(\max y)$ :

(i) $\hat{\varphi}(\max y)=\{i\}$ and therefore $\varphi(y)=i$ as $l>1$ (when $l=1$, the case $\varphi(y)=\tilde{0}$ is also possible), or

(ii) $\hat{\varphi}(\max y)=\{1, \ldots, l\}$ and hence $\varphi(y)=\tilde{0}$ as $l<n$, or

(iii) $\hat{\varphi}(\max y)=\{1, \ldots, n\}$ and thus $\varphi(y)=0$ (when $l=n$, the case $\varphi(y)=\tilde{0}$ is also possible).

The results of $\S 2$ tell us that in order to consider the possibility of deleting a relation, $s$, from the set of all left-packed binary algebraic relations on $\underline{P}_{n}$, without destroying the duality, we must investigate the nature of these relations on the dual $D(\underline{s})$ of the test algebra $\underline{s}$.

Let $r$ and $s$ be relations of shapes $k$ and $l$ respectively (maximal in $\alpha^{-1}(\leq)$ but no necessarily left-packed) with $1<l<n$. We shall see that even though $r$ is not itself the graph of a partial map on $P_{n}$ (since $\left.(d, d),(d, T) \in r\right)$, its interpretation on $D(\underline{s})$ is the graph of a partial map. We continue to represent $D(\underline{s})=\mathbf{B}_{n}\left(\underline{s}, \underline{P}_{n}\right)$ as $\mathscr{Y}_{n}\left(V_{n}, Y_{l}\right)$. Assume that $r$ is determined by the jointly 
surjective $p$-morphisms $\rho_{1}, \rho_{2}: V_{n} \rightarrow Y_{k}$. Thus $\rho_{1}(0)=0, \rho_{2}(0)=\tilde{0}$ and the top of $\rho_{1}$ is a permutation of $\{1, \ldots, n\}$. Consequently,

$$
\pi^{r}:=\hat{\rho}_{1}^{-1} \circ \hat{\rho}_{2}:\{1, \ldots, n\} \rightarrow\{1, \ldots, n\}
$$

is well defined. The map $\pi^{r}$ encodes the labels attached to the maximals above $\tilde{0}$ in $Y_{k}$ by the maps $\rho_{1}$ and $\rho_{2}$. Indeed, there is a point above $\tilde{0}$ in $Y_{l}$ to which $\rho_{1}$ and $\rho_{2}$ attach the labels $i$ and $\tilde{j}$ if and only if $\pi^{r}(j)=i$. We refer to the labels attached by $\rho_{1}$ to the maximals above $\tilde{0}$ in $Y_{k}$ as the left labels of $r$ and denote the set by $L_{r}$; thus

$$
L_{r}:=\rho_{1}^{-1}(\{1, \ldots, k\})=\pi^{r}(\{1, \ldots, n\}) .
$$

Finally, for all $X \in \mathscr{X}$ define the domain of $r$ on $X$ to be the set of all $x_{1} \in X$ such that $\left(x_{1}, x_{2}\right) \in r$ for some $x_{2} \in X$, and denote it by $\operatorname{dom} r$. By Proposition 3.3, a $p$-morphism $\varphi_{1}: V_{n} \rightarrow Y_{l}$ is in the domain of $r$ on $\mathscr{Y}_{n}\left(V_{n}, Y_{l}\right)$ if and only if $\varphi_{1}$ factors through $\rho_{1}$, say $\varphi_{1}=\mu \circ \rho_{1}$ for some $p$-morphism $\mu: Y_{k} \rightarrow Y_{l}$, in which case $\left(\varphi_{1}, \varphi_{2}\right) \in r$ where $\varphi_{2}:=\mu \circ \rho_{2}$. Since $\hat{\mu}=\hat{\varphi}_{1} \circ \hat{\rho}_{1}^{-1}$, it follows that the top of $\mu$ is uniquely determined by $\varphi_{1}$ and the top of $\varphi_{2}$ is given by

$$
\hat{\varphi}_{2}=\hat{\mu} \circ \hat{\rho}_{2}=\hat{\varphi}_{1} \circ \hat{\rho}_{1}^{-1} \circ \hat{\rho}_{2}=\hat{\varphi}_{1} \circ \pi^{r} .
$$

Thus, by Lemma 5.2, if $1<l<n$, the relation $r$ on $\mathscr{Y}_{n}\left(V_{n}, Y_{l}\right)$ is the graph of a partial map from dom $r$ into $\mathscr{Y}_{n}\left(V_{n}, Y_{l}\right)$.

Lemma 5.3. Let $r$ and $s$ be binary algebraic relations on $\underline{P}_{n}$ which are maximal in $\alpha^{-1}(\leq)$ and have shapes $k$ and $l$ respectively.

(i) If $k=1$, then every $p$-morphism $\varphi_{1}: V_{n} \rightarrow Y_{l}$ is in the domain of $r$ on $\mathscr{Y}_{n}\left(V_{n}, Y_{l}\right)$.

(ii) Let $1<k<n$ and let $\varphi_{1}: V_{n} \rightarrow Y_{l}$ be a p-morphism. Then $\varphi_{1}$ is in the domain of $r$ on $\mathscr{Y}_{n}\left(V_{n}, Y_{l}\right)$ if and only if

(a) $\varphi_{1}$ is the constant map $\kappa_{i}$ onto $\{i\}$ for some $i$ with $1 \leq i \leq n$, or

(b) $l=1$ and $\varphi_{1}\left(V_{n}\right)=\{\tilde{0}, 1\}$, or

(c) $1<l \leq n-k+1$ and $\left|\varphi_{1}\left(L_{r}\right)\right|=1$, or

(d) $1<l \leq k$ and $\varphi_{1}\left(L_{r}\right)=\{1, \ldots, l\}$.

(iii) If $k=n$, then a p-morphism $\varphi_{1}: V_{n} \rightarrow Y_{l}$ is in the domain of $r$ on $\mathscr{Y}_{n}\left(V_{n}, Y_{l}\right)$ if and only if

(a) $\varphi_{1}$ is the constant map $\kappa_{i}$ for some $i$ with $1 \leq i \leq n$, or

(b) $l=1$ and $\varphi_{1}\left(V_{n}\right)=\{\tilde{0}, 1\}$, or

(c) $1<l<n$ and $\varphi_{1}(\{1, \ldots, n\})=\{1, \ldots, l\}$, or

(d) $\varphi_{1}(\{1, \ldots, n\})=\{1, \ldots, n\}$ (regardless of the value of $l$ ).

(iv) Let $1<l<n$. Then, on $D(\underline{s})=\mathscr{Y}_{n}\left(V_{n}, Y_{l}\right)$, the relation $r$ is the graph of a partial map, also denoted by $r$, determined by $r\left(\widehat{\varphi_{1}}\right)=\hat{\varphi}_{1} \circ \pi^{r}$ for all $\varphi_{1}$ in the domain of $r$ on $\mathscr{Y}_{n}\left(V_{n}, Y_{l}\right)$.

Proof. The observations preceding the lemma prove (iv). If $\varphi_{1}=\mu \circ \rho_{1}$, then $\mu(0)=\mu\left(\rho_{1}(0)\right)=\varphi_{1}(0)$. It follows that $\varphi_{1}: V_{n} \rightarrow Y_{l}$ will be in the domain of $r$ precisely when we can choose $j \in Y_{l}$ such that

$$
\mu(0):=\varphi_{1}(0), \quad \mu(\tilde{0}):=j, \quad \text { and } \quad \hat{\mu}:=\hat{\varphi}_{1} \circ \hat{\rho}_{1}^{-1}
$$


defines a $p$-morphism $\mu: Y_{k} \rightarrow Y_{l}$. Before we consider Claims (i)-(iii) in turn, note that the constant map $\kappa_{i}$ is always in the domain of $r$-simply choose $\mu: Y_{k} \rightarrow Y_{l}$ to be the constant map onto $\{i\}$.

Claim (i). If $k=1$, then choosing $\mu(\tilde{0})=\hat{\mu}(1)$ in (*) yields the required $p$-morphism $\mu: Y_{k} \rightarrow Y_{l}$.

Claim (ii). Let $1<k<n$. Assume that $\varphi_{1}$ is in the domain of $r$ on $\mathscr{Y}_{n}\left(V_{n}, Y_{l}\right)$ and let $\mu: Y_{k} \rightarrow Y_{l}$ be a $p$-morphism with $\varphi_{1}=\mu \circ \rho_{1}$. Then

$$
\varphi_{1}\left(L_{r}\right)=\hat{\varphi}_{1} \circ \hat{\rho}_{1}^{-1}(\{1, \ldots, k\})=\hat{\mu}(\{1, \ldots, k\})=\mu(\max \tilde{0})=\max \mu(\tilde{0}) .
$$

As $k<n$, we have $|\max \mu(\tilde{0})| \in\{1, l\}$, whence $\left|\varphi_{1}\left(L_{r}\right)\right|=1$ or $\varphi_{1}\left(L_{r}\right)=$ $\{1, \ldots, l\}$. If $\left|\varphi_{1}\left(L_{r}\right)\right|=1$, then either

(a) $\varphi_{1}$ is a constant map on $\{i\}$ for some $i$ with $1 \leq i \leq n$, or

(b) $\hat{\varphi}_{1}$ is constant but $\varphi_{1}$ is not, in which case $l=1$ and $\varphi_{1}\left(V_{n}\right)=\{\tilde{0}, 1\}$, or

(c) $\hat{\varphi}_{1}$ is nonconstant, in which case $1<l$ and $\varphi_{1}(\{1, \ldots, n\})=\{1, \ldots, l\}$ whence $n-k \geq l-1$, i.e., $1<l \leq n-k+1$.

If $\left|\varphi_{1}\left(L_{r}\right)\right|>1$, then

(d) $\varphi_{1}\left(L_{r}\right)=\{1, \ldots, l\}$ and hence $1<l \leq k$ as $\left|L_{r}\right|=k$.

Conversely, in each of the Cases (a)-(d) it is easy to choose a value for $\mu(\tilde{0})$ so that $(*)$ above defines a $p$-morphism $\mu: Y_{k} \rightarrow Y_{l}$. If $\varphi_{1}=\kappa_{i}$, then define $\mu(\tilde{0})=i$; if $l=1$ and $\varphi_{1}\left(V_{n}\right)=\{\tilde{0}, 1\}$, then choose $\mu(\tilde{0}) \in\{\tilde{0}, 1\}$; if $1<l \leq n-k+1$ and $\left|\varphi_{1}\left(L_{r}\right)\right|=1$, then define $\mu(\tilde{0})=\hat{\mu}(1)$; if $1<l \leq k$ and $\varphi_{1}\left(L_{r}\right)=\{1, \ldots, l\}$, then define $\mu(\tilde{0})=\tilde{0}$.

Claim (iii). Let $k=n$. This is argued as in (ii), the only modifications required arising from the fact that we now have $|\max \mu(\tilde{0})| \in\{1, l, n\}$. For the converse, we define $\mu(\tilde{0})$ in Cases (a)-(d) as indicated below.

(a) If $\mu_{1}=\kappa_{i}$, then define $\mu(\tilde{0})=i$.

(b) Choose $\mu(\tilde{0}) \in\{\tilde{0}, 1\}$.

(c) Define $\mu(\tilde{0})=\tilde{0}$.

(d) Define $\mu(\tilde{0})=0$ if $1 \leq l<n$, and define $\mu(\tilde{0})=\tilde{0}$ if $l=n$ and $\varphi_{1}(0)=\tilde{0}$; choose $\mu(\tilde{0}) \in\{0, \tilde{0}\}$ if $l=n$ and $\varphi_{1}(0)=0$.

Lemma 5.4. Let $r$ and $s$ be left-packed relations of shape $l$ with $1<l<n$. Then there exists a binary algebraic relation $t$ on $\underline{P}_{n}$ of shape $l+1$ such that $\pi^{s}=\pi^{r} \circ \pi^{t}$. Moreover,

$$
(a, b) \in r \&(b, c) \in t \Rightarrow(a, c) \in s,
$$

that is, $r \circ t \subseteq s$, on $P_{n}$.

Proof. Let the partitions of $\{1, \ldots, n\}$ associated with $r$ and $s$ be $X_{1}, \ldots, X_{l}$ and $Y_{1}, \ldots, Y_{l}$ and let the pairs of jointly surjective $p$-morphisms associated with $r$ and $s$ be $\rho_{1}, \rho_{2}: V_{n} \rightarrow Y_{l}$ and $\sigma_{1}, \sigma_{2}: V_{n} \rightarrow Y_{l}$, respectively. Define $\tau_{1}, \tau_{2}: V_{n} \rightarrow Y_{l+1}$ by

$$
\begin{array}{lll}
\tau_{1}(1)=1 & \text { and } & \tau_{1}\left(\max X_{i}\right)=i+1 \quad \text { for } i=1, \ldots, l \\
\tau_{2}(1)=1 & \text { and } \quad \tau_{2}(i)=j+1 \quad \text { if } i \in Y_{j},
\end{array}
$$


and extend $\tau_{1}$ to a permutation on $\{1, \ldots, n\}$; further, define $\tau_{1}(0)=0$ and $\tau_{2}(0)=\tilde{0}$. Let $t$ be the binary algebraic relation on $\underline{P}_{n}$ corresponding to $\tau_{1}, \tau_{2}$. Thus

$$
\pi^{t}(i)=\hat{\tau}_{1}^{-1}\left(\hat{\tau}_{2}(i)\right)= \begin{cases}1 & \text { if } i=1, \\ \max X_{j} & \text { if } i \neq 1 \text { and } i \in Y_{j} .\end{cases}
$$

Since $r$ and $s$ are left-packed we have $\hat{\rho}_{1}=\hat{\sigma}_{1}=\mathrm{id}$, and hence $\pi^{r}=\rho_{2}$ and $\pi^{s}=\sigma_{2}$. Thus

$$
\begin{aligned}
\pi^{r}\left(\pi^{t}(i)\right) & = \begin{cases}1 & \text { if } i=1, \\
\rho_{2}\left(\max X_{j}\right) & \text { if } i \neq 1 \text { and } i \in Y_{j},\end{cases} \\
& = \begin{cases}1 & \text { if } i=1, \\
j & \text { if } i \neq 1 \text { and } i \in Y_{j},\end{cases} \\
& =\sigma_{2}(i)=\pi^{s}(i) .
\end{aligned}
$$

Hence $\pi^{r} \circ \pi^{t}=\pi^{s}$, as required.

As a subalgebra of $\underline{P}_{n}^{2}$, we have

$$
r=\left\{\left(\varphi \circ \rho_{1}, \varphi \circ \rho_{2}\right) \mid \varphi \in \mathbf{P}\left(Y_{l}, \underset{\approx}{\mathbf{2}}\right)\right\}
$$

Similarly,

$$
s=\left\{\left(\eta \circ \sigma_{1}, \eta \circ \sigma_{2}\right) \mid \eta \in \mathbf{P}\left(Y_{l}, \underset{\approx}{\mathbf{2}}\right)\right\}
$$

and

$$
t=\left\{\left(\psi \circ \tau_{1}, \psi \circ \tau_{2}\right) \mid \psi \in \mathbf{P}\left(Y_{l+1}, \underset{\approx}{\mathbf{2}}\right)\right\}
$$

Let $\varphi \in \mathbf{P}\left(Y_{l}, \underset{\approx}{\mathbf{2}}\right)$ and $\psi \in \mathbf{P}\left(Y_{l+1}, \underset{\approx}{\mathbf{2}}\right)$ with $\varphi \circ \rho_{2}=\psi \circ \tau_{1}$. Thus $a, b, c \in$ $P_{n}$ with $(a, b) \in r$ and $(b, c) \in t$, where

$$
a=\varphi \circ \rho_{1}, \quad b=\varphi \circ \rho_{2}=\psi \circ \tau_{1}, \quad c=\psi \circ \tau_{2} .
$$

In order to show that $(a, c) \in s$, we need $\eta \in \mathbf{P}\left(Y_{l}, \underset{\approx}{2}\right)$ such that

$$
\eta \circ \sigma_{1}=a=\varphi \circ \varphi_{1} \text { and } \eta \circ \sigma_{2}=c=\psi \circ \tau_{2}
$$

These equations force $\hat{\eta}=\hat{\varphi}$ as $\hat{\sigma}_{1}=\hat{\rho}_{1}=\mathrm{id}$. The first equation also yields $\eta(0)=\varphi(0)$ while the second yields $\eta(\tilde{0})=\psi(\tilde{0})$. Thus $\eta$ is uniquely determined by $\varphi$ and $\psi$. It remains to prove that $\eta$ is order-preserving given that $\varphi$ and $\psi$ are.

Note that $\varphi \circ \rho_{2}=\psi \circ \tau_{1}$ implies that $\varphi(\tilde{0})=\psi(0)$. Hence if $\varphi(0)=1$, then $\psi(0)=\varphi(\tilde{0})=1$. In this case $\eta=\varphi=\psi$. To prove that $\eta$ is order-preserving, it remains to prove that if $\varphi(i)=0$ for some $i$ with $1 \leq i \leq l$, then $\psi(\tilde{0})=0$.

For $1 \leq i \leq l$, we have

$$
\psi(i+1)=\psi\left(\tau_{1}\left(\tau_{1}^{-1}(i+1)\right)\right)=\varphi\left(\rho_{2}\left(\max X_{i}\right)\right)=\varphi(i) .
$$

Hence, if $\varphi(i)=0$, then $\psi(i+1)=0$ and thus $\psi(\tilde{0})=0$, as required.

Lemma 5.5. Let $r$ and $s$ be distinct left-packed relations of shape $l$ with $1<$ $l<n$ and let $t$ be a binary algebraic relation (not necessarily left-packed) of shape $k>l$ such that $\pi^{s}=\pi^{r} \circ \pi^{t}$. Let $\varphi_{1}, \varphi_{2} \in \mathscr{Y}_{n}\left(V_{n}, Y_{l}\right)$ with $\left(\varphi_{1}, \varphi_{2}\right) \in s$. Then there exists $\varphi_{3} \in \mathscr{Y}_{n}\left(V_{n}, Y_{l}\right)$ with $\left(\varphi_{1}, \varphi_{3}\right) \in r$ and $\left(\varphi_{3}, \varphi_{2}\right) \in t$.

Proof. Since $1<l<n$, the relations $r, s$, and $t$ can be regarded as partial functions on $\mathscr{Y}_{n}\left(V_{n}, Y_{l}\right)$, by Lemma 5.3 (iv). 
Thus the required conclusion can be rewritten as: "If $\varphi_{1} \in \mathscr{Y}_{n}\left(V_{n}, Y_{l}\right)$ with $\varphi_{1} \in \operatorname{dom} s$, then $\varphi_{1} \in \operatorname{dom} r, r\left(\varphi_{1}\right) \in \operatorname{dom} t$ and $t\left(r\left(\varphi_{1}\right)\right)=s\left(\varphi_{1}\right)$."

Let $\varphi_{1} \in \mathscr{Y}\left(V_{n}, Y_{l}\right)$ with $\varphi_{1} \in \operatorname{dom} s$. Since $r$ and $s$ are left-packed, $r$ and $s$ have the same sets of left labels; indeed, $L_{r}=L_{s}=\{1, \ldots, l\}$. Thus, by Lemma 5.3(ii), the relations $r$ and $s$ have the same domain, whence $\varphi_{1} \in \operatorname{dom} r$. Note that

$$
t\left(r\left(\varphi_{1}\right)\right)^{-}=r \widehat{\left(\varphi_{1}\right)} \circ \pi^{t}=\hat{\varphi}_{1} \circ \pi^{r} \circ \pi^{t}=\hat{\varphi}_{1} \circ \pi^{s}=s \widehat{\left(\varphi_{1}\right)},
$$

and hence $t\left(r\left(\varphi_{1}\right)\right)=s\left(\varphi_{1}\right)$ (by Lemma 5.2) provided we can show that $r\left(\varphi_{1}\right) \in$ dom $t$. Now consider the four subcases of Lemma 5.3(ii).

Case (a). If $\varphi_{1}$ is the constant map $\kappa_{i}$, then $r\left(\kappa_{i}\right)=\kappa_{i} \in \operatorname{dom} t$, as required.

Case (b). This case does not arise as $1<l$.

Case (c). If $\varphi_{1}\left(L_{r}\right)=\{i\}$, then

$$
r\left(\varphi_{1}\right)(\{1, \ldots, n\})=\varphi_{1}\left(\pi^{r}(\{1, \ldots, n\})\right)=\varphi_{1}\left(L_{r}\right)=\{i\} .
$$

Since $1<l$, it follows that $r\left(\varphi_{1}\right)=\kappa_{i} \in \operatorname{dom} t$.

$$
\begin{aligned}
& \text { Case }(\mathrm{d}) \text {. If } \varphi_{1}\left(L_{r}\right) \\
& \qquad \begin{aligned}
r\left(\varphi_{1}\right)\left(L_{t}\right) & =\left(\hat{\varphi}_{1} \circ \pi^{r}\right)\left(L_{t}\right)=\left(\hat{\varphi}_{1} \circ \pi^{r}\right)\left(\pi^{t}(\{1, \ldots, n\})\right) \\
& =\left(\hat{\varphi}_{1} \circ \pi^{r} \circ \pi^{t}\right)(\{1, \ldots, n\}) \\
& =\left(\hat{\varphi}_{1} \circ \pi^{s}\right)(\{1, \ldots, n\}) \text { as } \pi^{s}=\pi^{r} \circ \pi^{t} \\
& =\hat{\varphi}_{1}\left(L_{s}\right)=\{1, \ldots, l\} .
\end{aligned}
\end{aligned}
$$

Thus, if $k<n$, then $r\left(\varphi_{1}\right) \in \operatorname{dom} t$ by Lemma 5.3(ii)(d). Also if $k=n$, then $r\left(\varphi_{1}\right) \in \operatorname{dom} t$ by Lemma 5.3(iii)(c),(d).

Lemma 5.6. Let $1<l<n$ and let $r$ be a left-packed relation on $\underline{P}_{n}$ of shape $l$. If $\Phi: D(\underline{r}) \rightarrow P_{n}$ preserves $r$ and also preserves every binary algebraic relation on $\underline{P}_{n}$ of shape $k$ with $k>l$, then $\Phi$ preserves every left-packed relation on $\underline{P}_{n}$ of shape $l$.

Proof. As usual, we represent $D(\underline{r})$ as $\mathscr{Y}_{n}\left(V_{n}, Y_{l}\right)$. Let $\varphi_{1}, \varphi_{2} \in \mathscr{Y}_{n}\left(V_{n}, Y_{l}\right)$ with $\left(\varphi_{1}, \varphi_{2}\right) \in s$, where $s$ is another left-packed relation of shape $l$. By Lemmas 5.3 and 5.4, there exists a (not necessarily left-packed) relation $t$ of shape $l+1$ and $\varphi_{3} \in \mathscr{Y}_{n}\left(V_{n}, Y_{l}\right)$ such that $\left(\varphi_{1}, \varphi_{3}\right) \in r$ and $\left(\varphi_{3}, \varphi_{2}\right) \in t$. By hypothesis, we have $\left(\Phi\left(\varphi_{1}\right), \Phi\left(\varphi_{2}\right)\right) \in r$ and $\left(\Phi\left(\varphi_{3}\right), \Phi\left(\varphi_{2}\right)\right) \in t$ on $P$. But, since $\pi^{s}=\pi^{r} \circ \pi^{t}$, this forces $\left(\Phi\left(\varphi_{1}\right), \Phi\left(\varphi_{2}\right)\right) \in s$, as required.

This final lemma yields an inductive proof of the second half of Theorem 5.1; the first half is just a restatement of Theorem 4.4.

Proof of Theorem 5.1. In the proof of Theorem 3.6 in [4], is shown that for every relation $r$ of shape $l$, there is a left-packed relation $r^{\prime}$ of shape $l$ such that $\left\{r^{\prime}\right\} \cup G$ generates $r$ and $\{r\} \cup G$ generates $r^{\prime}$. Hence, without loss of generality, we may assume that every member of $S$ is left-packed.

For $1 \leq m \leq n$, let $S^{(m)}$ consist of precisely one left-packed relation of shape $k$ for $1 \leq k \leq m$ and all relations of shape $k$ for $m<k \leq n$. By Proposition 3.2, if $S$ is the set of all left-packed relations, then $S \cup G$ yields a duality on $\mathbf{B}_{n}$. Since there is only one left-packed relation of shape 1, it follows $S^{(1)} \cup G$ yields a duality on $\mathbf{B}_{n}$. Now let $1<l<n$ and assume that 
$S^{(l-1)} \cup G$ yields a duality on $\mathbf{B}_{n}$. Lemma 5.6 implies that $S^{(l)}$ generates each relation $s \in S^{(l-1)} \backslash S^{(l)}$ on the test algebra $\underline{s}=K\left(Y_{l}\right)$. Hence, by Proposition 2.4, $S^{(l)} \cup G$ yields a duality on $\mathbf{B}_{n}$. Thus, by induction, $S^{(n-1)} \cup G$ yields a duality on $\mathbf{B}_{n}$, which is the desired result as $S^{(n-1)}$ contains precisely $n$ leftpacked relations, one of each shape, as there is only one left-packed relation of shape $n$.

\section{REFERENCES}

1. B. A. Davey, Topological duality for prevarieties of universal algebras, Studies in Foundations and Combinatorics, Advances in Mathematics: Supplementary Studies 1, Academic Press, New York, 1978, pp. 61-99.

2. _ Dualities for Stone algebras, double Stone algebras, and relative Stone algebras, Colloq. Math. 46 (1982), 1-14.

3. B. A. Davey and H. A. Priestley, Generalised piggyback dualities and applications to Ockham algebras, Houston J. Math. 13 (1987), 151-198.

4. __ Partition-induced natural dualities for varieites of pseudocomplemented distributive lattices, Discrete Math. (to appear).

5. B. A. Davey and H. Werner, Dualities and equivalences for varieties of algebras, Colloq. Math. Soc. János Bolyai 33 (1983), 101-275.

6. ___ Piggyback dualities, Colloq. Math. Soc. János Bolyai 43 (1986), 61-83.

7. __ Piggyback-dualitäten, Bull. Austral. Math. Soc. 32 (1985), 1-32.

8. G. Grätzer, Lattice theory: first concepts and distributive lattices, Freeman, San Francisco, Calif., 1971.

9. H. P. Gumm, Geometrical methods in congruence modular algebras, Mem. Amer. Math. Soc. No. 286, 1983.

10. H. A. Priestley, The construction of spaces dual to pseudocomplemented distributive lattices, Quart J. Math. Oxford Ser. (2) 26 (1975), 215-228.

11. H. A. Priestley and M. P. Ward, A multi-purpose backtracking algorithm.

12. M. H. Stone, The theory of representations for Boolean algebras, Trans. Amer. Math. Soc. 40 (1936), 37-111.

13. M. P. Ward, D. Phil. thesis, University of Oxford, 1989.

Mathematics, La Trobe University, Bundoora, Victoria 3083, Australia

E-mail address: davey@latcs1.oz.au

Mathematical Institute, 24/29 St. Giles, Oxford OX1 3LB, England

E-mail address: hap@vax.oxford.ac.uk 